\title{
Asymptotic Expansions for Stationary Distributions of Nonlinearly Perturbed Semi-Markov Processes. 2
}

\author{
Dmitrii Silvestrov $^{1} \cdot$ Sergei Silvestrov $^{2}$
}

Received: 17 December 2016 / Revised: 9 October 2017 /

Accepted: 12 October 2017 / Published online: 18 November 2017

(C) The Author(s) 2017. This article is an open access publication

\begin{abstract}
Asymptotic expansions with explicit upper bounds for remainders are given for stationary distributions of nonlinearly perturbed semi-Markov processes with finite phase spaces. The corresponding algorithms are based on a special technique of sequential phase space reduction, which can be applied to processes with an arbitrary asymptotic communicative structure of phase spaces.
\end{abstract}

Keywords Markov chain · Semi-Markov process · Nonlinear perturbation · Stationary distribution $\cdot$ Expected hitting time $\cdot$ Laurent asymptotic expansion

Mathematics Subject Classification (2010) 60J10 - 60J27 · 60K15 · 65C40

\section{Introduction}

This is Part 2 of the paper, Silvestrov and Silvestrov (2017). In what follows, we continue counting of sections, lemmas, theorems and relations from Part 1.

In Part 1, algorithms for constructing of asymptotic expansions, with remainders of a standard form $o(\cdot)$, for stationary distributions of nonlinearly perturbed semi-Markov processes have been given. In Part 2, we present algorithms for construction the above asymptotic expansions in a more advanced form, with explicit upper bounds for remainders.

Dmitrii Silvestrov

silvestrov@math.su.se

Sergei Silvestrov

sergei.silvestrov@mdh.se

1 Department of Mathematics, Stockholm University, Stockholm, Sweden

2 Division of Applied Mathematics, School of Education, Culture and Communication, Mälardalen University, Västerås, Sweden 
We consider models, where the phase space is one class of communicative states, for embedded Markov chains of pre-limiting perturbed semi-Markov processes, while it can possess an arbitrary communicative structure, i.e., can consist of one or several closed classes of communicative states and, possibly, a class of transient states, for the limiting embedded Markov chain.

The initial perturbation conditions are formulated in the forms of Taylor and Laurent asymptotic expansions with explicit upper bounds for remainders, respectively, for transition probabilities (of embedded Markov chains) and expectations of sojourn times, for perturbed semi-Markov processes.

The algorithms are based on special time-space screening procedures for sequential phase space reduction and algorithms for re-calculation of asymptotic expansions with explicit upper bounds for remainders, which constitute perturbation conditions for the semi-Markov processes with reduced phase spaces.

The final asymptotic expansions for stationary distributions of nonlinearly perturbed semi-Markov processes are given in the form of Taylor asymptotic expansions, with explicit upper bounds for remainders.

The algorithms presented in the paper have an universal character. They can be applied to perturbed semi-Markov processes with an arbitrary asymptotic communicative structure and are computationally effective due to recurrent character of computational procedures.

The short survey of works in the area is given in the introduction to Part 1 of this paper. A comprehensive bibliography of works in the area can be found in the recent paper by Silvestrov and Silvestrov (2016a).

Part 2 of the paper includes six sections and two appendices.

In Section 8, we present explicit formulas for computing parameters of explicit power upper bounds remainders of expansions obtained as results of multiplication by constant, summation, multiplication and division operations with asymptotic Laurent expansions (Lemmas 9-12).

In Section 9, we get Laurent asymptotic expansions with explicit upper bounds for remainders for transition characteristics of nonlinearly perturbed semi-Markov processes. The method is based on re-computing of asymptotic expansions and upper bounds for remainders for transition characteristics of perturbed semi-Markov processes (obtained by application of the time-space procedure of one-state reduction of phase space described in Section 9 and the above operational rules for Laurent asymptotic expansions) via the corresponding expansions for transition characteristics of initial semi-Markov processes (Theorems 6 and 7).

In Section 10, we present recurrent algorithms for computing asymptotic expansions with explicit upper bounds for remainders for expected hitting times of nonlinearly perturbed reduced semi-Markov processes, obtained as the result of sequential repetition of the above one-step time-space screening procedure of one-state reduction of phase space (Theorem 8).

In Section 11, we, finally, get asymptotic expansions with explicit upper bounds for remainders for stationary distributions of nonlinearly perturbed reduced semi-Markov processes (Theorem 9). This theorem presents results which can be interpreted as high order analogs for results concerned explicit upper estimates for rates of convergence in simple asymptotic relations about convergence of stationary probabilities to their limiting values, for perturbed semi-Markov processes. Such upper estimates are replaced by asymptotic expansions for stationary probabilities with explicit upper bounds for remainders, in Theorem 9. 
In Appendix A, we give short proofs of Lemmas 1-4 and 9-12, which present operational rules for Laurent asymptotic expansions.

In Appendix B, we present an example illustrating theoretical results obtained in the present paper.

We would like to conclude the introduction with the remark that the present paper is a shorten version of the report Silvestrov and Silvestrov (2016b), where one can find some additional details of proofs, comments and references.

\section{Laurent Asymptotic Expansions with Explicit Upper Bounds for Remainders}

In this section, we present so-called operational rules for Laurent asymptotic expansions with explicit upper bounds for remainders. The corresponding proofs and comments are given in Appendix A.

Let $A(\varepsilon)$ be a real-valued function defined on an interval $\left(0, \varepsilon_{0}\right]$, for some $0<\varepsilon_{0} \leq 1$, and given on this interval by a Laurent asymptotic expansion,

$$
A(\varepsilon)=a_{h_{A}} \varepsilon^{h_{A}}+\cdots+a_{k_{A}} \varepsilon^{k_{A}}+o_{A}\left(\varepsilon^{k_{A}}\right),
$$

where (a) $-\infty<h_{A} \leq k_{A}<\infty$ are integers, (b) coefficients $a_{h_{A}}, \ldots, a_{k_{A}}$ are real numbers, (c) $\left|o_{A}\left(\varepsilon^{k_{A}}\right)\right| \leq G_{A} \varepsilon^{k_{A}+\delta_{A}}$, for $0<\varepsilon \leq \varepsilon_{A}$, where (d) $0<\delta_{A} \leq 1,0 \leq G_{A}<\infty$ and $0<\varepsilon_{A} \leq \varepsilon_{0}$.

We refer to such Laurent asymptotic expansion as a $\left(h_{A}, k_{A}, \delta_{A}, G_{A}, \varepsilon_{A}\right)$-expansion.

The $\left(h_{A}, k_{A}, \delta_{A}, G_{A}, \varepsilon_{A}\right)$-expansion is also a $\left(h_{A}, k_{A}\right)$-expansion, according to the definition given in Part I of the paper, since, $o_{A}\left(\varepsilon^{k_{A}}\right) / \varepsilon^{k_{A}} \rightarrow 0$ as $\varepsilon \rightarrow 0$.

We say that $\left(h_{A}, k_{A}, \delta_{A}, G_{A}, \varepsilon_{A}\right)$-expansion $A(\varepsilon)$ is pivotal if it is known that $a_{h_{A}} \neq 0$.

It is useful to note that there is no sense to consider, it seems, a more general case of upper bounds for the remainder $o_{A}\left(\varepsilon^{k_{A}}\right)$, with parameter $\delta_{A}>1$. Indeed, let us define $k_{A}^{\prime}=k_{A}+\left[\delta_{A}\right]-\mathrm{I}\left(\delta_{A}=\left[\delta_{A}\right]\right)$ and $\delta_{A}^{\prime}=\delta_{A}-\left[\delta_{A}\right]+\mathrm{I}\left(\delta_{A}=\left[\delta_{A}\right]\right) \in(0,1]$. The $\left(h_{A}, k_{A}, \delta_{A}, G_{A}, \varepsilon_{A}\right)$-expansion $A(\varepsilon)$ can be re-written in the equivalent form of the $\left(h_{A}, k_{A}^{\prime}, \delta_{A}^{\prime}, G_{A}, \varepsilon_{A}\right)$-expansion, $A(\varepsilon)=a_{h_{A}} \varepsilon^{h_{A}}+\cdots+a_{k_{A}} \varepsilon^{k_{A}}+0 \varepsilon^{k_{A}+1}+\cdots+0 \varepsilon^{k_{A}^{\prime}}+$ $o_{A}^{\prime}\left(\varepsilon^{k_{A}^{\prime}}\right)$, with the remainder $o_{A}^{\prime}\left(\varepsilon^{k_{A}^{\prime}}\right)=o_{A}\left(\varepsilon^{k_{A}}\right)$, which satisfies inequalities $\left|o_{A}^{\prime}\left(\varepsilon^{k_{A}^{\prime}}\right)\right|=$ $\left|o_{A}\left(\varepsilon^{k_{A}}\right)\right| \leq G_{A} \varepsilon^{k_{A}+\delta_{A}}=G_{A} \varepsilon^{k_{A}^{\prime}+\delta_{A}^{\prime}}$, for $0<\varepsilon \leq \varepsilon_{A}$.

The following proposition supplements Lemma 1 .

Lemma 9 If $A(\varepsilon)=a_{h_{A}^{\prime}}^{\prime} \varepsilon^{h_{A}^{\prime}}+\cdots+a_{k_{A}^{\prime}}^{\prime} \varepsilon^{k_{A}^{\prime}}+o_{A}^{\prime}\left(\varepsilon^{k_{A}^{\prime}}\right)=a_{h_{A^{\prime \prime}}^{\prime \prime}} \varepsilon^{h_{A^{\prime \prime}}}+\cdots+a_{k_{A^{\prime \prime}}}{ }^{\prime \prime} \varepsilon^{k_{A^{\prime \prime}}}+$ $o_{A^{\prime \prime}}\left(\varepsilon^{k_{A}^{\prime \prime}}\right), \varepsilon \in\left(0, \varepsilon_{0}\right]$ can be represented as, respectively, $\left(h_{A}^{\prime}, k_{A}^{\prime}, \delta_{A}^{\prime}, G_{A}^{\prime}, \varepsilon_{A}^{\prime}\right)$ - and $\left(h_{A}^{\prime \prime}, k_{A}^{\prime \prime}, \delta_{A}^{\prime \prime}, G_{A}^{\prime \prime}, \varepsilon_{A}^{\prime \prime}\right)$-expansion, then the $\left(h_{A}, k_{A}\right)$-expansion $A(\varepsilon)=a_{h_{A}} \varepsilon^{h_{A}}+\cdots+$ $a_{k_{A}} \varepsilon^{k}+o_{A}\left(\varepsilon^{k_{A}}\right), \varepsilon \in\left(0, \varepsilon_{0}\right]$ given in Lemma 1 is an $\left(h_{A}, k_{A}, \delta_{A}, G_{A}, \varepsilon_{A}\right)$-expansion, with parameters $G_{A}, \delta_{A}$ and $\varepsilon_{A}$ given by the following formulas:

(a) $\delta_{A}=\delta_{A}^{\prime} \wedge \delta_{A}^{\prime \prime}$;

(b) $G_{A}=G_{A}^{\prime} \vee G_{A}^{\prime \prime}$;

(c) $\varepsilon_{A}=\varepsilon_{A}^{\prime} \wedge \varepsilon_{A}^{\prime \prime}$.

It is also useful to mention that a constant $a$ can be interpreted as function $A(\varepsilon) \equiv a$. Thus, 0 can be represented, for any integer $-\infty<h \leq k<\infty$, as the $\left(h, k, \delta_{h, k}, G_{h, k}\right.$, $\varepsilon_{h, k}$ )-expansion, $0=0 \varepsilon^{h}+\ldots+0 \varepsilon^{k}+o\left(\varepsilon^{k}\right)$, with remainder $o\left(\varepsilon^{k}\right) \equiv 0$ and, thus, parameters 
$\delta_{h, k}=1, G_{h, k}=0$, and $\varepsilon_{h, k}=\varepsilon_{0}$. Also, 1 can be represented, for any integer $0 \leq k<\infty$, as the $\left(0, k, \delta_{k}, G_{k}, \varepsilon_{k}\right)$-expansion, $1=1+0 \varepsilon+\ldots+0 \varepsilon^{k}+o\left(\varepsilon^{k}\right)$, with remainder $o\left(\varepsilon^{k}\right) \equiv 0$ and, thus, parameters $\delta_{k}=1, G_{k}=0$, and $\varepsilon_{k}=\varepsilon_{0}$.

Let us consider four Laurent asymptotic expansions, $A(\varepsilon)=a_{h_{A}} \varepsilon^{h_{A}}+\cdots+a_{k_{A}} \varepsilon^{k_{A}}+$ $o_{A}\left(\varepsilon^{k_{A}}\right), B(\varepsilon)=b_{h_{B}} \varepsilon^{h_{B}}+\cdots+b_{k_{B}} \varepsilon^{k_{B}}+o_{B}\left(\varepsilon^{k_{B}}\right), C(\varepsilon)=c_{h_{C}} \varepsilon^{h_{C}}+\cdots+c_{k_{C}} \varepsilon^{k_{C}}+o_{C}\left(\varepsilon^{k_{C}}\right)$, and $D(\varepsilon)=d_{h_{D}} \varepsilon^{h_{D}}+\cdots+d_{k_{D}} \varepsilon^{k_{D}}+o_{D}\left(\varepsilon^{k_{D}}\right)$ defined for $0<\varepsilon \leq \varepsilon_{0}$, for some $0<\varepsilon_{0} \leq 1$.

Let us denote, $F_{A}=\max _{h_{A} \leq i \leq k_{A}}\left|a_{i}\right|, F_{B}=\max _{h_{B} \leq i \leq k_{B}}\left|b_{i}\right|, F_{C}=\max _{h_{C} \leq i \leq k_{C}}\left|c_{i}\right|$ and $F_{D}=\max _{h_{D} \leq i \leq k_{D}}\left|d_{i}\right|$.

The following lemma presents operational rules for computing parameters of upper bounds for remainders of Laurent asymptotic expansions.

Lemma 10 The above asymptotic expansions have the following operational rules for computing remainders:

(i) If $A(\varepsilon), \varepsilon \in\left(0, \varepsilon_{0}\right]$ is a $\left(h_{A}, k_{A}, \delta_{A}, G_{A}, \varepsilon_{A}\right)$-expansion and $c$ is a constant, then $C(\varepsilon)=c A(\varepsilon), \varepsilon \in\left(0, \varepsilon_{0}\right]$ is a $\left(h_{C}, k_{C}, \delta_{C}, G_{C}, \varepsilon_{C}\right)$-expansion with parameters $h_{C}, k_{C}$ and coefficients $c_{r}, r=h_{C}, \ldots, k_{C}$ given in proposition (i) of Lemma 2 , and parameters $\delta_{C}, G_{C}$ and $\varepsilon_{C}$ given by the formulas:
(a) $\delta_{C}=\delta_{A}$;
(b) $G_{C}=|c| G_{A}$;
(c) $\varepsilon_{C}=\varepsilon_{A}$.

(ii) If $A(\varepsilon), \varepsilon \in\left(0, \varepsilon_{0}\right]$ is a $\left(h_{A}, k_{A}, \delta_{A}, G_{A}, \varepsilon_{A}\right)$-expansion and $B(\varepsilon), \varepsilon \in\left(0, \varepsilon_{0}\right]$ is $a\left(h_{B}, k_{B}, \delta_{B}, G_{B}, \varepsilon_{B}\right)$-expansion, then $C(\varepsilon)=A(\varepsilon)+B(\varepsilon), \varepsilon \in\left(0, \varepsilon_{0}\right]$ is a $\left(h_{C}, k_{C}, \delta_{C}, G_{C}, \varepsilon_{C}\right)$-expansion with parameters $h_{C}, k_{C}$ and coefficients $c_{r}, r=$ $h_{C}, \ldots, k_{C}$ given in proposition (ii) of Lemma 2, and parameters $\delta_{C}, G_{C}$ and $\varepsilon_{C}$ given by formulas:
(a) $\delta_{C}=\delta_{A} \wedge \delta_{B}$;
(b) $G_{C}=F_{A}\left(k_{A}-k_{C}\right)+G_{A}+F_{B}\left(k_{B}-k_{C}\right)+G_{B}$;
(c) $\varepsilon_{C}=\varepsilon_{A} \wedge \varepsilon_{B}$.

(iii) If $A(\varepsilon), \varepsilon \in\left(0, y_{0}\right]$ is a $\left(h_{A}, k_{A}, \delta_{A}, G_{A}, \varepsilon_{A}\right)$-expansion and $B(\varepsilon), \varepsilon \in\left(0, \varepsilon_{0}\right]$ is $a\left(h_{B}, k_{B}, \delta_{B}, G_{B}, \varepsilon_{B}\right)$-expansion, then $C(\varepsilon)=A(\varepsilon) \cdot B(\varepsilon), \varepsilon \in\left(0, \varepsilon_{0}\right]$ is a $\left(h_{C}, k_{C}, \delta_{C}, G_{C}, \varepsilon_{C}\right)$-expansion with parameters $h_{C}, k_{C}$ and coefficients $c_{r}, r=$ $h_{C}, \ldots, k_{C}$ given in proposition (iii) of Lemma 2 , and parameters $\delta_{C}, G_{C}$ and $\varepsilon_{C}$ given by formulas:
(a) $\delta_{C}=\delta_{A} \wedge \delta_{B}$;
(b) $G_{C}=\left(F_{A}\left(k_{A}-h_{A}+1\right)+G_{A}\right)\left(F_{B}\left(k_{B}-h_{B}+1\right)+G_{B}\right)$;
(c) $\varepsilon_{C}=\varepsilon_{A} \wedge \varepsilon_{B}$.

(iv) If $B(\varepsilon), \varepsilon \in\left(0, \varepsilon_{0}\right]$ is a pivotal $\left(h_{B}, k_{B}, \delta_{B}, G_{B}, \varepsilon_{B}\right)$-expansion, then there exists $\varepsilon_{C} \leq \varepsilon_{0}^{\prime} \leq \varepsilon_{0}$ such that $B(\varepsilon) \neq 0, \varepsilon \in\left(0, \varepsilon_{0}^{\prime}\right]$, and $C(\varepsilon)=\frac{1}{B(\varepsilon)}, \varepsilon \in\left(0, \varepsilon_{0}^{\prime}\right]$ is a pivotal $\left(h_{C}, k_{C}, \delta_{C}, G_{C}, \varepsilon_{C}\right)$-expansion with parameters $h_{C}, k_{C}$ and coefficients $c_{r}, r=h_{C}, \ldots, k_{C}$ given in proposition (iv) of Lemma 2 , and parameters $\delta_{C}, G_{C}$ and $\varepsilon_{C}$ given formulas:
(a) $\delta_{C}=\delta_{B}=\tilde{\delta}_{C}$;
(b) $G_{C}=\left(\frac{\left|b_{h_{B}}\right|}{2}\right)^{-1}\left(F_{B}\left(k_{B}-h_{B}+1\right)+G_{B}\right) F_{C}\left(k_{C}-h_{C}+1\right)$;
(c) $\varepsilon_{C}=\varepsilon_{B} \wedge \tilde{\varepsilon}_{B}$, where $\tilde{\varepsilon}_{B}=\left(\frac{\left|b_{h_{B}}\right|}{2\left(F_{B}\left(k_{B}-h_{B}\right)+G_{B}\right)}\right)^{\frac{1}{\delta_{B}}}$. 
(v) If $A(\varepsilon), \varepsilon \in\left(0, \varepsilon_{0}\right]$ is a $\left(h_{A}, k_{A}, \delta_{A}, G_{A}, \varepsilon_{A}\right)$-expansion, $B(\varepsilon), \varepsilon \in\left(0, \varepsilon_{0}\right]$ is a pivotal $\left(h_{B}, k_{B}, \delta_{B}, G_{B}, \varepsilon_{B}\right)$-expansion, then there exists $\varepsilon_{D} \leq \varepsilon_{0}^{\prime} \leq \varepsilon_{0}$ such that $B(\varepsilon) \neq 0, \varepsilon \in\left(0, \varepsilon_{0}^{\prime}\right]$, and $D(\varepsilon)=\frac{A(\varepsilon)}{B(\varepsilon)}$ is a $\left(h_{D}, k_{D}, \delta_{D}, G_{D}, \varepsilon_{D}\right)$-expansion with parameters $h_{D}, k_{D}$ and coefficients $d_{r}, r=h_{D}, \ldots, k_{D}$ given in proposition (v) of Lemma 2, and parameters $\delta_{D}, G_{D}, \varepsilon_{D}$ given by formulas:

(a) $\delta_{D}=\delta_{A} \wedge \delta_{B}$;

(b) $G_{D}=\left(\frac{\left|b_{h_{B}}\right|}{2}\right)^{-1}\left(F_{A}\left(k_{A}-k_{A} \wedge\left(h_{A}+k_{B}-h_{B}\right)\right)+G_{A}+F_{B} F_{D}\left(k_{B}-h_{B}+\right.\right.$ 1) $\left.\left(k_{D}-h_{D}+1\right)+G_{B} F_{D}\left(k_{D}-h_{D}+1\right)\right)$;

(c) $\varepsilon_{D}=\varepsilon_{A} \wedge \varepsilon_{B} \wedge \tilde{\varepsilon}_{B}$, where $\tilde{\varepsilon}_{B}$ is given in relation (c) of proposition (iv).

Let $A_{m}(\varepsilon)=a_{h_{A_{m}}, m} \varepsilon^{h_{A_{m}}}+\cdots+a_{k_{A_{m}}, m} \varepsilon^{k_{A_{m}}}+o_{A_{m}}\left(\varepsilon^{k_{A_{m}}}\right), \varepsilon \in\left(0, \varepsilon_{0}\right]$ be a $\left(h_{A_{m}}, k_{A_{m}}\right.$, $\delta_{A_{m}}, G_{A_{m}}, \varepsilon_{A_{m}}$ )-expansion, for $m=1, \ldots, N$.

Also, let us denote $F_{A_{m}}=\max _{h_{A_{m}} \leq i \leq k_{A_{m}}}\left|a_{i, m}\right|$, for $m=1, \ldots, N$.

Lemma 11 The above asymptotic expansions have the following multiple operational rules for computing remainders:

(i) $B_{n}(\varepsilon)=A_{1}(\varepsilon)+\cdots+A_{n}(\varepsilon), \varepsilon \in\left(0, \varepsilon_{0}\right]$ is, for every $n=1, \ldots, N, a\left(h_{B_{n}}, k_{B_{n}}\right.$, $\left.\delta_{B_{n}}, G_{B_{n}}, \varepsilon_{B_{n}}\right)$-expansion, with parameters $h_{B_{n}}, k_{B_{n}}, n=1, \ldots, N$ and coefficients $b_{h_{B_{n}}+l, n}, l=0, \ldots, k_{B_{n}}-h_{B_{n}}, n=1, \ldots, N$ given in proposition $(i)$ of Lemma 3 , and parameters $G_{B_{n}}, \delta_{B_{n}}, y_{B_{n}}, n=1, \ldots, N$ given by formulas:

(a) $\delta_{B_{n}}=\min _{1 \leq m \leq n} \delta_{A_{m}}$;

(b) $G_{B_{n}}=\sum_{1 \leq i \leq n}\left(F_{A_{i}}\left(k_{A_{i}}-k_{B_{n}}\right)+G_{A_{i}}\right)$;

(c) $\varepsilon_{B_{n}}=\min _{1 \leq i \leq n} \varepsilon_{A_{i}}$.

(ii) $C_{n}(\varepsilon)=A_{1}(\varepsilon) \times \cdots \times A_{n}(\varepsilon), \varepsilon \in\left(0, \varepsilon_{0}\right]$ is, for $n=1, \ldots, N$, $a\left(h_{C_{n}}, k_{C_{n}}, \delta_{C_{n}}\right.$, $G_{C_{n}}, \varepsilon_{C_{N}}$ )-expansion with parameters $h_{C_{n}}, k_{C_{n}}, n=1, \ldots, N$ and coefficients $c_{h_{C_{n}}+l, n}, l=0, \ldots, k_{C_{n}}-h_{C_{n}}, n=1, \ldots, N$ given in proposition (ii) of Lemma 3 , and parameters $G_{C_{n}}, \delta_{C_{n}}, \varepsilon_{C_{n}}, n=1, \ldots, N$ given by formulas:

(a) $\delta_{C_{n}}=\min _{1 \leq m \leq n} \delta_{A_{m}}$;

(b) $G_{C_{n}}=\prod_{1 \leq m \leq n}\left(F_{A_{m}}\left(k_{A_{m}}-h_{A_{m}}+1\right)+G_{A_{m}}\right)$.

(c) $\varepsilon_{C_{n}}=\min _{1 \leq i \leq n} \varepsilon_{A_{i}}$.

(iii) Parameters $\delta_{B_{n}}, G_{B_{n}}, \varepsilon_{B_{n}}, n=1, \ldots, N$ and $\delta_{C_{n}}, G_{C_{n}}, \varepsilon_{C_{n}}, n=1, \ldots, N$ in upper bounds for remainders in the asymptotic expansions, respectively, for functions $B_{n}(\varepsilon)=A_{1}(\varepsilon)+\cdots+A_{n}(\varepsilon), n=1, \ldots, N$ and $C_{n}(\varepsilon)=A_{1}(\varepsilon) \times \cdots \times A_{n}(\varepsilon), n=$ $1, \ldots, N$ are invariant with respect to any permutation, respectively, of summation and multiplication order in the above formulas.

The summation and multiplication rules for computing of upper bounds for remainders given in propositions (ii) and (iii) of Lemma 10 possess the communicative property, but do not possess the associative and distributional properties.

Lemma 10 let us get an effective low bound for parameter $\delta_{A}$ for any $\left(h_{A}, k_{A}, \delta_{A}\right.$, $G_{A}, \varepsilon_{A}$ )-expansion $A(\varepsilon)$ obtained as the result of a finite sequence of operations (described in Lemma 10) performed over expansions from some finite set of such expansions.

The following lemma summarize these properties of Laurent asymptotic expansions with explicit upper bounds for remainders. 
Lemma 12 The summation and multiplication operations for Laurent asymptotic expansions defined in Lemma 2 possess the following algebraic properties, which should be understood as equalities for the corresponding parameters of upper bounds for their remainders:

(i) The functional identity, $C(\varepsilon) \equiv A(\varepsilon)+B(\varepsilon) \equiv B(\varepsilon)+A(\varepsilon)$, implies that $\delta_{C}=$ $\delta_{A+B}=\delta_{B+A}, G_{C}=G_{A+B}=G_{B+A}$ and $\varepsilon_{C}=\varepsilon_{A+B}=\varepsilon_{B+A}$.

(ii) The functional identity, $C(\varepsilon) \equiv A(\varepsilon) \cdot B(\varepsilon) \equiv B(\varepsilon) \cdot A(\varepsilon)$, implies that $\delta_{C}=\delta_{A \cdot B}=$ $\delta_{B \cdot A}, G_{C}=G_{A \cdot B}=G_{B \cdot A}$ and $\varepsilon_{C}=\varepsilon_{A \cdot B}=\varepsilon_{B} \cdot A$.

(iii) If $A(\varepsilon)$ is $\left(h_{A}, k_{A}, \delta_{A}, G_{A}, \varepsilon_{A}\right)$-expansion obtained as the result of a finite sequence of operations (multiplication by a constant, summation, multiplication, and division) performed over $\left(h_{A_{i}}, k_{A_{i}}, \delta_{A_{i}}, G_{A_{i}}, \varepsilon_{A_{i}}\right)$-expansions $A_{i}(\varepsilon), i=1, \ldots, N$, according to the rules presented in Lemmas 2 and 10 , then $\delta_{A} \geq \delta_{N}^{*}=\min _{1 \leq i \leq N} \delta_{A_{i}}$. This makes it possible to rewrite $A(\varepsilon)$ as the $\left(h_{A}, k_{A}, \delta_{N}^{*}, G_{A, N}^{*}, \varepsilon_{A}\right)$-expansion, with parameter $G_{A, N}^{*}=G_{A} \varepsilon_{A}^{\delta_{A}-\delta_{N}^{*}}$.

\section{Asymptotic Expansions with Explicit Upper Bounds for Remainders for Transition Characteristics}

Let us recall the perturbed semi-Markov processes $\eta^{(\varepsilon)}(t), t \geq 0$, with phase space $\mathbb{X}=$ $\{1, \ldots, N\}$ and transition probabilities $Q_{i j}^{(\varepsilon)}(t), t \geq 0, i, j \in \mathbb{X}$, introduced in Part I of the paper. These processes depend on a perturbation parameter $\varepsilon \in\left(0, \varepsilon_{0}\right]$, for some $0<\varepsilon_{0} \leq$ 1. We also recall transition probabilities of the corresponding embedded Markov chains, $p_{i j}(\varepsilon)=Q_{i j}^{(\varepsilon)}(\infty), i, j \in \mathbb{X}$, and expectations of sojourn times $e_{i j}(\varepsilon)=\int_{0}^{\infty} t Q_{i j}^{(\varepsilon)}(d t)$, $i, j \in \mathbb{X}$.

We assume that condition $\mathbf{A}$, introduced in Part I, holds for semi-Markov processes $\eta^{(\varepsilon)}(t)$. In particular, we recall the transition sets $\mathbb{Y}_{i}, i \in \mathbb{X}$ (which include states $j \in \mathbb{X}$ with non-zero probabilities $p_{i j}(\varepsilon)$ and guarantee ergodicity of the processes $\left.\eta^{(\varepsilon)}(t)\right)$ introduced in this condition.

However, we replace the perturbation condition $\mathbf{D}$ by the following stronger condition, in which the corresponding Taylor asymptotic expansions are given in the form with explicit upper bounds for remainders:

$\mathbf{D}^{\prime}: \quad p_{i j}(\varepsilon)=\sum_{l=l_{i j}^{-}}^{l_{i j}^{+}} a_{i j}[l] \varepsilon^{l}+o_{i j}\left(\varepsilon^{l_{i j}^{+}}\right), \varepsilon \in\left(0, \varepsilon_{0}\right]$, for $j \in \mathbb{Y}_{i}, i \in \mathbb{X}$, where (a) $0 \leq$ $l_{i j}^{-} \leq l_{i j}^{+}<\infty$ are integers, coefficients $a_{i j}[k], l_{i j}^{-} \leq k \leq l_{i j}^{+}$are real numbers, and $a_{i j}\left[l_{i j}^{-}\right]>0$, for $j \in Y_{i}, i \in X$; (b) $\left|o_{i j}\left(\varepsilon^{l_{i j}^{+}}\right)\right| \leq G_{i j} \varepsilon^{l_{i j}^{+}+\delta_{i j}}, 0<\varepsilon \leq \varepsilon_{i j}$, for $j \in \mathbb{Y}_{i}, i \in \mathbb{X}$, where $0<\delta_{i j} \leq 1,0 \leq G_{i j}<\infty$ and $0<\varepsilon_{i j} \leq \varepsilon_{0}$.

Also, we replace the perturbation condition $\mathbf{E}$ by the following stronger condition, in which the corresponding Laurent asymptotic expansions are given in the form with explicit upper bounds for remainders:

$\mathbf{E}^{\prime}: \quad e_{i j}(\varepsilon)=\sum_{l=m_{i j}^{-}}^{m_{i j}^{+}} b_{i j}[l] \varepsilon^{l}+\dot{o}_{i j}\left(\varepsilon^{m_{i j}^{+}}\right), \varepsilon \in\left(0, \varepsilon_{0}\right]$, for $j \in \mathbb{Y}_{i}, i \in \mathbb{X}$, where (a) $-\infty<m_{i j}^{-} \leq m_{i j}^{+}<\infty$ are integers, coefficients $b_{i j}[l], m_{i j}^{-} \leq l \leq m_{i j}^{+}$are real numbers, and $b_{i j}\left[m_{i j}^{-}\right]>0$, for $j \in \mathbb{Y}_{i}, i \in \mathbb{X}$; (b) $\left|\dot{o}_{i j}\left(\varepsilon^{m_{i j}^{+}}\right)\right| \leq \dot{G}_{i j} \varepsilon^{m_{i j}^{+}+\dot{\delta}_{i j}}, 0<$ $\varepsilon \leq \dot{\varepsilon}_{i j}$, for $j \in \mathbb{Y}_{i}, i \in \mathbb{X}$, where $0<\dot{\delta}_{i j} \leq 1,0 \leq \dot{G}_{i j}<\infty$ and $0<\dot{\varepsilon}_{i j} \leq \varepsilon_{0}$. 
We would like to re-call the comments given in Section 10, which explain slightly unusual formulations of lemmas and theorems, which includes references to descriptions of algorithms given in their proofs.

As was pointed out in Part I, condition A implies that sets $\mathbb{Y}_{r r}^{+}=\mathbb{Y}_{r r} \backslash\{r\} \neq \emptyset, r \in \mathbb{X}$ and the non-absorption probability $\bar{p}_{r r}(\varepsilon)=1-p_{r r}(\varepsilon) \in(0,1]$, for $r \in \mathbb{X}, \varepsilon \in\left(0, \varepsilon_{0}\right]$. This probability satisfy the following relation, for every $r \in \mathbb{X}, \varepsilon \in\left(0, \varepsilon_{0}\right]$,

$$
\bar{p}_{r r}(\varepsilon)=1-p_{r r}(\varepsilon)=\sum_{j \in \mathbb{Y}_{r r}^{+}} p_{r j}(\varepsilon) .
$$

The above relation let us construct an algorithm for getting asymptotic expansions with explicit upper bounds for remainders, for non-absorption probabilities $\bar{p}_{r r}(\varepsilon)$.

Lemma 13 Let conditions $\boldsymbol{A}$ and $\boldsymbol{D}$, hold. Then, for every $r \in \mathbb{X}$, the pivotal $\left(\bar{l}_{r r}^{-}, \bar{l}_{r r}^{+}\right)$expansion for the non-absorption probability $\bar{p}_{r r}(\varepsilon)$ given in Lemma $8_{*}$ is, also, a $\left(\bar{l}_{r r}^{-}, \bar{l}_{r r}^{+}, \bar{\delta}_{r r}, \bar{G}_{r r}, \bar{\varepsilon}_{r r}\right)$-expansion, with parameters $\bar{\delta}_{r r}, \bar{G}_{r r}$ and $\bar{\varepsilon}_{r r}$, which can be computed according to the algorithm described below, in the proof of the lemma.

Proof Let $r \in \mathbb{Y}_{r}$. First, propositions (i) of Lemmas 3 and 11 (the multiple summation rule) should be applied to the sum $\sum_{j \in \mathbb{Y}_{r r}^{+}} p_{r j}(\varepsilon)$. Second, propositions (i) (the multiplication by constant -1 ) and (ii) (the summation with constant 1) of Lemmas 2 and 10 should be applied to the asymptotic expansion for probability $p_{r r}(\varepsilon)$ given in condition $\mathbf{D}^{\prime}$, in order to get the asymptotic expansion for function $1-p_{r r}(\varepsilon)$. Third, Lemmas 1 and 9 should be applied to the asymptotic expansion for function $\bar{p}_{r r}(\varepsilon)$ given in two alternative forms by relation (31). This yields the corresponding pivotal the $\left(\bar{l}_{r r}^{-}, \bar{l}_{r r}^{+}\right)$-expansion for probabilities $\bar{p}_{r r}(\varepsilon)$, given in Lemma 8, and proves that this expansion is a $\left(\bar{l}_{r r}^{-}, \bar{l}_{r r}^{+}, \bar{\delta}_{r r}, \bar{G}_{r r}, \bar{\varepsilon}_{r r}\right)$-expansion, with parameters computed in the process of realization of the above algorithm. The case $r \notin \mathbb{Y}_{r}$ is trivial, since, in this case, probability $\bar{p}_{r r}(\varepsilon) \equiv 1$.

Let us recall formula (19) for the transition probabilities ${ }_{r} p_{i j}(\varepsilon), i, j \in{ }_{r} \mathbb{X}=\mathbb{X} \backslash\{r\}$ of the reduced embedded Markov chain ${ }_{r} \eta_{n}^{(\varepsilon)}$, introduced in Part I,

$$
{ }_{r} p_{i j}(\varepsilon)=p_{i j}(\varepsilon)+p_{i r}(\varepsilon) \frac{p_{r j}(\varepsilon)}{1-p_{r r}(\varepsilon)} .
$$

Let us introduce parameter, $\delta^{\circ}=\min _{j \in \mathbb{Y}_{i}, i \in \mathbb{X}} \delta_{i j}$. Obviously, inequalities $\delta_{i j} \geq \delta^{\circ}, j \in$ $\mathbb{Y}_{i}, i \in \mathbb{X}$ hold for parameters $\delta_{i j}$ appearing in upper bounds for the remainders of asymptotic expansions in condition $\mathbf{D}^{\prime}$.

Theorem 6 Conditions $\boldsymbol{A}$ and $\boldsymbol{D}^{\prime}$, assumed to hold for the Markov chains $\eta_{n}^{(\varepsilon)}$, also hold for the reduced Markov chains ${ }_{r} \eta_{n}^{(\varepsilon)}$, for every $r \in \mathbb{X}$. Also, for every $j \in{ }_{r} \mathbb{Y}_{i}, i \in{ }_{r} \mathbb{X}, r \in \mathbb{X}$, the pivotal $\left({ }_{r} l_{i j}^{-},{ }_{r} l_{i j}^{+}\right)$-expansion for the transition probability ${ }_{r} p_{i j}(\varepsilon)$ given in Theorem 2 is $a\left({ }_{r} l_{i j}^{-},{ }_{r} l_{i j}^{+},{ }_{r} \delta_{i j},{ }_{r} G_{i j},{ }_{r} \varepsilon_{i j}\right)$-expansion appearing in condition $\boldsymbol{D}^{\prime}$ for the Markov chains ${ }_{r} \eta_{n}^{(\varepsilon)}$. Parameters ${ }_{r} \delta_{i j},{ }_{r} G_{i j}$ and ${ }_{r} \varepsilon_{i j}$ can be computed using the algorithm described below, in the proof of the theorem. The inequalities ${ }_{r} \delta_{i j} \geq \delta^{\circ}, j \in{ }_{r} \mathbb{Y}_{i}, i \in{ }_{r} \mathbb{X}, r \in \mathbb{X}$ hold.

Proof Condition A holds for the Markov chains ${ }_{r} \eta_{n}^{(\varepsilon)}$ by Lemma 6, with the same parameter $\varepsilon_{0}$ as for the Markov chains $\eta_{n}^{(\varepsilon)}$ and with the transition sets ${ }_{r} \mathbb{Y}_{i}, i \in r \mathbb{X}$ given by relation (20). 
Let us prove that condition $\mathbf{D}^{\prime}$ holds for the Markov chains ${ }_{r} \eta_{n}^{(\varepsilon)}$, with the same parameter $\varepsilon_{0}$ as for the Markov chains $\eta_{n}^{(\varepsilon)}$ and the transition sets ${ }_{r} \mathbb{Y}_{i}, i \in{ }_{r} \mathbb{X}$ given by relation (20). Let $j, r \in \mathbb{Y}_{i} \cap \mathbb{Y}_{r}$. First, propositions (v) (the division rule) of Lemmas 2 and 10 should be applied to the quotient $\frac{p_{r j}(\varepsilon)}{1-p_{r r}(\varepsilon)}$. Second, propositions (iii) (the multiplication rule) of Lemmas 2 and 10 should be applied to the product $p_{i r}(\varepsilon) \cdot \frac{p_{r j}(\varepsilon)}{1-p_{r r}(\varepsilon)}$. Third, propositions (ii) (the summation rule) of Lemmas 2 and 10 should be applied to $\operatorname{sum}_{r} p_{i j}(\varepsilon)=$ $p_{i j}(\varepsilon)+p_{i r}(\varepsilon) \cdot \frac{p_{r j}(\varepsilon)}{1-p_{r r}(\varepsilon)}$. The asymptotic expansions for probabilities $p_{i r}(\varepsilon), p_{r j}(\varepsilon)$, and $p_{i j}(\varepsilon)$, given in condition $\mathbf{D}^{\prime}$, and probability $1-p_{r r}(\varepsilon)$, given in Lemmas 8 and 13 , should be used. This yields the corresponding pivotal $\left({ }_{r} l_{i j}^{-},{ }_{r} l_{i j}^{+}\right)$-expansions for transition probabilities ${ }_{r} p_{i j}(\varepsilon), j \in{ }_{\mathbb{Y}}, i \in{ }_{r} \mathbb{X}, r \in \mathbb{X}$, given in Theorem 2, and proves that these expansions are $\left({ }_{r} l_{i j}^{-},{ }_{r} l_{i j}^{+},{ }_{r} \delta_{i j},{ }_{r} G_{i j},{ }_{r} \varepsilon_{i j}\right)$-expansions, with parameters computed in the process of realization of the above algorithm. If $j \notin \mathbb{Y}_{i}$ then $p_{i j}(\varepsilon) \equiv 0$; if $j \notin \mathbb{Y}_{r}$ then $p_{r j}(\varepsilon) \equiv 0$; if $r \notin \mathbb{Y}_{i}$ then $p_{i r}(\varepsilon) \equiv 0$; if $r \notin \mathbb{Y}_{r}$ then $1-p_{r r}(\varepsilon) \equiv 1$. In these cases, the above algorithm is readily simplified. Thus, condition $\mathbf{D}^{\prime}$ holds for the reduced Markov chains $r \eta_{n}^{(\varepsilon)}$.

Inequalities ${ }_{r} \delta_{i j} \geq \delta^{\circ}, j \in{ }_{r} \mathbb{Y}_{i}, i \in r \mathbb{X}, r \in \mathbb{X}$ hold, by proposition (iii) of Lemma 12 .

Let us recall formula (22) for the expectations of sojourn times ${ }_{r} e_{i j}(\varepsilon)$, for $i, j \in{ }_{r} \mathbb{X}=$ $\mathbb{X} \backslash\{r\}$ for the reduced semi-Markov process ${ }_{r} \eta^{(\varepsilon)}(t)$, introduced in Part I,

$$
\begin{aligned}
{ }_{r} e_{i j}(\varepsilon)= & e_{i j}(\varepsilon)+e_{i r}(\varepsilon) \frac{p_{r j}(\varepsilon)}{1-p_{r r}(\varepsilon)} \\
& +e_{r r}(\varepsilon) \frac{p_{i r}(\varepsilon)}{1-p_{r r}(\varepsilon)} \frac{p_{r j}(\varepsilon)}{1-p_{r r}(\varepsilon)}+e_{r j}(\varepsilon) \frac{p_{i r}(\varepsilon)}{1-p_{r r}(\varepsilon)} .
\end{aligned}
$$

Let us introduce parameter, $\delta^{*}=\min _{j \in \mathbb{Y}_{i}, i \in \mathbb{X}}\left(\delta_{i j} \wedge \dot{\delta}_{i j}\right)$. Obviously, inequalities $\delta_{i j}, \dot{\delta}_{i j} \geq \delta^{*}, j \in \mathbb{Y}_{i}, i \in \mathbb{X}$ hold for parameters $\delta_{i j}$ and $\dot{\delta}_{i j}$ appearing in upper bounds for the remainders of asymptotic expansions in conditions $\mathbf{D}^{\prime}$ and $\mathbf{E}^{\prime}$.

Theorem 7 Conditions $\boldsymbol{A}-\boldsymbol{C}, \boldsymbol{D}^{\prime}$ and $\boldsymbol{E}^{\prime}$, assumed to hold for the semi-Markov processes $\eta^{(\varepsilon)}(t)$, also hold for the reduced semi-Markov processes ${ }_{r} \eta^{(\varepsilon)}(t)$, for every $r \in \mathbb{X}$. Also, for every $j \in{ }_{r} \mathbb{Y}_{i}, i \in{ }_{r} \mathbb{X}, r \in \mathbb{X}$, the pivotal $\left({ }_{r} m_{i j}^{-},{ }_{r} m_{i j}^{+}\right)$-expansion for expectation ${ }_{r} e_{i j}(\varepsilon)$ given in Theorem 3 is a $\left({ }_{r} m_{i j}^{-},{ }_{r} m_{i j}^{+},{ }_{r} \dot{\delta}_{i j},{ }_{r} \dot{G}_{i j},{ }_{r} \dot{\varepsilon}_{i j}\right)$-expansion appearing in condition $\boldsymbol{E}^{\prime}$ for the semi-Markov processes ${ }_{r} \eta^{(\varepsilon)}(t)$. Parameters ${ }_{r} \dot{\delta}_{i j},{ }_{r} \dot{G}_{i j}$ and ${ }_{r} \dot{\varepsilon}_{i j}$ can be computed using the algorithm described below, in the proof of the theorem. The inequalities ${ }_{r} \dot{\delta}_{i j} \geq$ $\delta^{*}, j \in r \mathbb{Y}_{i}, i \in r \mathbb{X}, r \in \mathbb{X}$ hold.

Proof Conditions $\mathbf{A}$ and $\mathbf{D}^{\prime}$ hold for the semi-Markov processes ${ }_{r} \eta^{(\varepsilon)}(t)$, respectively, by Lemma 6 and Theorem 6, with the same parameter $\varepsilon_{0}$ as for the semi-Markov processes $\eta^{(\varepsilon)}(t)$, and the transition sets ${ }_{r} \mathbb{Y}_{i}, i \in{ }_{r} \mathbb{X}$ given by relation (20). Also conditions $\mathbf{B}$ and $\mathbf{C}$ hold for processes $r \eta^{(\varepsilon)}(t)$, by Lemma 7 .

Let us prove that condition $\mathbf{E}^{\prime}$ holds for the semi-Markov processes ${ }_{r} \eta^{(\varepsilon)}(t)$, with the same parameter $\varepsilon_{0}$ and the transition sets $r \mathbb{Y}_{i}, i \in{ }_{r} \mathbb{X}$ given by relation (20). Let $j, r \in \mathbb{Y}_{i} \cap \mathbb{Y}_{r}$. First, propositions (v) (the division rule) of Lemmas 2 and 10 should be applied to the quotients $\frac{p_{r j}(\varepsilon)}{1-p_{r r}(\varepsilon)}$ and $\frac{p_{i r}(\varepsilon)}{1-p_{r r}(\varepsilon)}$. Second, propositions (iii) (the multiplication rule) of Lemmas 2 and 10 should be applied to the products $e_{i r}(\varepsilon) \cdot \frac{p_{r j}(\varepsilon)}{1-p_{r r}(\varepsilon)}$ and $e_{r j}(\varepsilon) \cdot \frac{p_{i r}(\varepsilon)}{1-p_{r r}(\varepsilon)}$ and 
propositions (ii) (the multiple multiplication rule) of Lemmas 3 and 11 to the product $e_{r r}(\varepsilon) \cdot \frac{p_{i r}(\varepsilon)}{1-p_{r r}(\varepsilon)} \cdot \frac{p_{r j}(\varepsilon)}{1-p_{r r}(\varepsilon)}$. Third, propositions (i) (the multiple summation rule) of Lemmas 3 and 11 should be applied to $\operatorname{sum}_{r} e_{i j}(\varepsilon)=e_{i j}(\varepsilon)+e_{i r}(\varepsilon) \cdot \frac{p_{r j}(\varepsilon)}{1-p_{r r}(\varepsilon)}+e_{r r}(\varepsilon) \cdot \frac{p_{i r}(\varepsilon)}{1-p_{r r}(\varepsilon)}$. $\frac{p_{r j}(\varepsilon)}{1-p_{r r}(\varepsilon)}+e_{r j}(\varepsilon) \cdot \frac{p_{i r}(\varepsilon)}{1-p_{r r}(\varepsilon)}$. The asymptotic expansions for probabilities $p_{i r}(\varepsilon), p_{i r}(\varepsilon)$ and $p_{i j}(\varepsilon)$, given in condition $\mathbf{D}^{\prime}$, probability $1-p_{r r}(\varepsilon)$, given in Lemmas 8 and 13 , and expectations $e_{i j}(\varepsilon), e_{i r}(\varepsilon), e_{r r}(\varepsilon)$ and $e_{r j}(\varepsilon)$, given in condition $\mathbf{E}^{\prime}$, should be used. This, first, yields the corresponding pivotal $\left(r m_{i j}^{-}, r m_{i j}^{+}\right)$-expansions for expectations of sojourn times ${ }_{r} e_{i j}(\varepsilon), j \in r \mathbb{Y}_{i}, i \in r \mathbb{X}, r \in \mathbb{X}$, given in Theorem 3, and, second, proves that these expansions are $\left({ }_{r} m_{i j}^{-},{ }_{r} m_{i j}^{+},{ }_{r} \dot{\delta}_{i j},{ }_{r} \dot{G}_{i j},{ }_{r} \dot{\varepsilon}_{i j}\right)$-expansions, with parameters computed in the process of realization of the above algorithm. If $j \notin \mathbb{Y}_{i}$ then $p_{i j}(\varepsilon) \equiv 0$ and $e_{i j}(\varepsilon) \equiv 0$; if $j \notin \mathbb{Y}_{r}$ then $p_{r j}(\varepsilon) \equiv 0$ and $e_{r j}(\varepsilon) \equiv 0$; if $r \notin \mathbb{Y}_{i}$ then $p_{i r}(\varepsilon) \equiv 0$ and $e_{i r}(\varepsilon) \equiv 0$; if $r \notin \mathbb{Y}_{r}$ then $1-p_{r r}(\varepsilon) \equiv 1$ and $e_{r r}(\varepsilon) \equiv 0$. In these cases, the above algorithm is readily simplified. Thus, condition $\mathbf{E}^{\prime}$ holds for the reduced semi-Markov processes $r \eta^{(\varepsilon)}(t)$.

Inequalities ${ }_{r} \dot{\delta}_{i j} \geq \delta^{*}, j \in{ }_{r} \mathbb{Y}_{i}, i \in{ }_{r} \mathbb{X}, r \in \mathbb{X}$ hold, by proposition (iii) of Lemma 12.

\section{Asymptotic Expansions with Explicit Upper Bounds for Remainders for Expected Hitting Times}

As in Part I, let $\bar{r}_{i, N}=\left\langle r_{i, 1}, \ldots, r_{i, N}\right\rangle=\left\langle r_{i, 1}, \ldots, r_{i, N-1}, i\right\rangle$ be a permutation of the sequence $\langle 1, \ldots, N\rangle$ such that $r_{i, N}=i$, and let $\bar{r}_{i, n}=\left\langle r_{i, 1}, \ldots, r_{i, n}\right\rangle, n=1, \ldots, N$ be the corresponding chain of growing sequences of states from space $\mathbb{X}$.

Theorem 8 Let conditions $\boldsymbol{A}-\boldsymbol{C}, \boldsymbol{D}^{\prime}$ and $\boldsymbol{E}^{\prime}$ hold for the semi-Markov processes $\eta^{(\varepsilon)}(t)$. Then, for every $i \in \mathbb{X}$, the pivotal $\left(M_{i i}^{-}, M_{i i}^{+}\right)$-expansion for the expectation of hitting time $E_{i i}(\varepsilon)$, given in Theorem 4 and obtained as the result of sequential exclusion of states $r_{i, 1}, \ldots, r_{i, N-1}$ from the phase space $\mathbb{X}$ of the processes $\eta^{(\varepsilon)}(t)$, is $a\left(M_{i i}^{-}, M_{i i}^{+}, \bar{r}_{i, N-1} \dot{\delta}_{i i}, \bar{r}_{i, N-1} \dot{G}_{i i}, \bar{r}_{i, N-1} \dot{\varepsilon}_{i i}\right)$-expansion. Parameters $\bar{r}_{i, N-1} \dot{\delta}_{i i}, \bar{r}_{i, N-1} \dot{G}_{i i}$ and $\bar{r}_{i, N-1} \dot{\varepsilon}_{i i}$ can be computed using the algorithm described below, in the proof of the theorem. Also, inequality $\bar{r}_{i, N-1} \dot{\delta}_{i i} \geq \delta^{*}$ holds making it possible to rewrite function $E_{i i}(\varepsilon)$ as the pivotal $\left(M_{i i}^{-}, M_{i i}^{+}, \delta^{*}, \bar{r}_{i, N-1} G_{i i}^{*}, \bar{r}_{i, N-1} \dot{\varepsilon}_{i i}\right)$-expansion, with parameter $\bar{r}_{i, N-1} G_{i i}^{*}=$ $\left.\bar{r}_{i, N-1} \dot{G}_{i i} \cdot\left(\bar{r}_{i, N-1} \dot{\varepsilon}_{i i}\right)^{\left(\bar{r}_{i, N-1}\right.} \dot{\delta}_{i i}-\delta^{*}\right)$.

Proof Let us assume that $p_{i}^{(\varepsilon)}=1$. Denote as $\bar{r}_{i, 0} \eta^{(\varepsilon)}(t)=\eta^{(\varepsilon)}(t)$ the initial semi-Markov process. Let us exclude state $r_{i, 1}$ from the phase space of semi-Markov process $\bar{r}_{i, 0} \eta^{(\varepsilon)}(t)$ using the time-space screening procedure described in Section 10. Let $\bar{r}_{i, 1} \eta^{(\varepsilon)}(t)$ be the corresponding reduced semi-Markov process. The above procedure can be repeated. The state $r_{i, 2}$ can be excluded from the phase space of the semi-Markov process $\bar{r}_{i, 1} \eta^{(\varepsilon)}(t)$. Let $\bar{r}_{i, 2} \eta^{(\varepsilon)}(t)$ be the corresponding reduced semi-Markov process. By continuing the above procedure for states $r_{i, 3}, \ldots, r_{i, n}$, we construct the reduced semi-Markov process $\bar{r}_{i, n} \eta^{(\varepsilon)}(t)$.

The process $\bar{r}_{i, n} \eta^{(\varepsilon)}(t)$ has the phase space $\bar{r}_{i, n} \mathbb{X}=\mathbb{X} \backslash\left\{r_{i, 1}, r_{i, 2}, \ldots, r_{i, n}\right\}$. The transition probabilities $\bar{r}_{i, n} p_{i^{\prime} j^{\prime}}(\varepsilon), i^{\prime}, j^{\prime} \in \bar{r}_{i, n} \mathbb{X}$ and the expectations of sojourn times $\bar{r}_{i, n} e_{i^{\prime} j^{\prime}}(\varepsilon), i^{\prime}, j^{\prime} \in \bar{r}_{i, n} \mathbb{X}$ are determined for the process $\bar{r}_{i, n} \eta^{(\varepsilon)}(t)$ by the transition probabilities and the expectations of sojourn times for the process $\bar{r}_{i, n-1} \eta^{(\varepsilon)}(t)$, via relations (32) and (33). 
By Theorem 1, the expectation of hitting time $E_{i^{\prime} j^{\prime}}(\varepsilon)$ coincides for the semi-Markov processes $\bar{r}_{i, 0} \eta^{(\varepsilon)}(t), \bar{r}_{i, 1} \eta^{(\varepsilon)}(t), \ldots, \bar{r}_{i, n} \eta^{(\varepsilon)}(t)$, for every $i^{\prime}, j^{\prime} \in \bar{r}_{i, n} \mathbb{X}$.

By Theorems 2, 3, 6 and 7, the semi-Markov processes $\bar{r}_{i, n} \eta^{(\varepsilon)}(t)$ satisfy conditions $\mathbf{A}-$ $\mathbf{C}, \mathbf{D}^{\prime}$ and $\mathbf{E}^{\prime}$. The transition sets $\bar{r}_{i, n} \mathbb{Y}_{i^{\prime}}, i^{\prime} \in \bar{r}_{i, n} \mathbb{X}$, for the process $\bar{r}_{i, n} \eta^{(\varepsilon)}(t)$, are determined by the transition sets $\bar{r}_{i, n-1} \mathbb{Y}_{i^{\prime}}, i^{\prime} \in \bar{r}_{i, n-1} \mathbb{X}$, for the process $\bar{r}_{i, n-1} \eta^{(\varepsilon)}(t)$, via relation (20). For every $j^{\prime} \in \bar{r}_{i, n} \mathbb{Y}_{i^{\prime}}, i^{\prime} \in \bar{r}_{i, n} \mathbb{X}$, the pivotal $\left(\bar{r}_{i, n} l_{i^{\prime} j^{\prime}}^{-}, \bar{r}_{i, n} l_{i^{\prime} j^{\prime}}^{+}\right)$-expansion for transition probability $\bar{r}_{i, n} p_{i^{\prime} j^{\prime}}(\varepsilon)$, given in Theorem 2, is, by Theorem 6, a $\left(\bar{r}_{i, n} l_{i^{\prime} j^{\prime}}^{-}, \bar{r}_{i, n} l_{i^{\prime} j^{\prime}}^{+}, \bar{r}_{i, n} \delta_{i^{\prime} j^{\prime}}, \bar{r}_{i, n} G_{i^{\prime} j^{\prime}}\right.$, $\bar{r}_{i, n} \varepsilon_{i^{\prime} j^{\prime}}$ )-expansion, with parameters $\bar{r}_{i, n} \delta_{i^{\prime} j^{\prime}}, \bar{r}_{i, n} G_{i^{\prime} j^{\prime}}$ and $\bar{r}_{i, n} \varepsilon_{i^{\prime} j^{\prime}}$ given in this theorem. Analogously, for every $j^{\prime} \in \bar{r}_{i, n} \mathbb{Y}_{i^{\prime}}, i^{\prime} \in \bar{r}_{i, n} \mathbb{X}$, the pivotal $\left(\bar{r}_{i, n} m_{i^{\prime} j^{\prime}}^{-}, \bar{r}_{i, n} m_{i^{\prime} j^{\prime}}^{+}\right)$-expansion for expectation $\bar{r}_{i, n} e_{i^{\prime} j^{\prime}}(\varepsilon)$, given in Theorem 3, is, by Theorem 7, a $\left(\bar{r}_{i, n} m_{i^{\prime} j^{\prime}}^{-}, \bar{r}_{i, n} m_{i^{\prime} j^{\prime}}^{+}, \bar{r}_{i, n} \dot{\delta}_{i^{\prime} j^{\prime}}\right.$, $\bar{r}_{i, n} \dot{G}_{i^{\prime} j^{\prime}, \bar{r}_{i, n}} \dot{\varepsilon}_{i^{\prime} j^{\prime}}$ )-expansion, with parameters $\bar{r}_{i, n} \dot{\delta}_{i^{\prime} j^{\prime}}, \bar{r}_{i, n} \dot{G}_{i^{\prime} j^{\prime}}$ and $\bar{r}_{i, n} \dot{\varepsilon}_{i^{\prime} j^{\prime}}$ given in this theorem. Also, by Theorem 7, the inequalities $\bar{r}_{i, n} \dot{\delta}_{i^{\prime} j^{\prime}} \geq \delta^{*}, j^{\prime} \in \bar{r}_{i, n} \mathbb{Y}_{i^{\prime}}, i^{\prime} \in \bar{r}_{i, n} \mathbb{X}$ hold.

The algorithm described above has a recurrent form and should be realized sequentially for the reduced semi-Markov processes $\bar{r}_{i, 1} \eta^{(\varepsilon)}(t), \ldots, \bar{r}_{i, n} \eta^{(\varepsilon)}(t)$ starting from the initial semi-Markov process $\bar{r}_{i, 0} \eta^{(\varepsilon)}(t)$.

Let us take $n=N-1$. The semi-Markov process $\bar{r}_{i, N-1} \eta^{(\varepsilon)}(t)$ has the phase space $\bar{r}_{i, N-1} \mathbb{X}=\mathbb{X} \backslash\left\{r_{i, 1}, r_{i, 2}, \ldots, r_{i, N-1}\right\}=\{i\}$, which is a one-state set. The process $\bar{r}_{i, N-1} \eta^{(\varepsilon)}(t)$ returns to state $i$ after every jump. Its transition probability $\bar{r}_{i, N-1} p_{i i}(\varepsilon)=1$, and the expectation of hitting time, $E_{i i}(\varepsilon)=\bar{r}_{i, N-1} e_{i i}(\varepsilon)$. This equality and Theorem 4 yield, for every $i \in$ $\mathbb{X}$, the pivotal $\left(M_{i i}^{-}, M_{i i}^{+}\right)$-expansion for expectation $E_{i i}(\varepsilon)$, which is invariant with respect to any permutation $\bar{r}_{i, N-1}^{\prime}=\left\langle r_{i, 1}^{\prime}, \ldots, r_{i, N-1}^{\prime}\right\rangle$ of the sequence $\bar{r}_{i, N-1}=\left\langle r_{i, 1}, \ldots, r_{i, N-1}\right\rangle$. This invariance also implies that parameters $\bar{r}_{i, N-1} m_{i i}^{ \pm}=M_{i i}^{ \pm}$do not depend on the choice of sequence $\bar{r}_{i, N-1}$. The above $\left(M_{i i}^{-}, M_{i i}^{+}\right)$-expansion for $E_{i i}(\varepsilon)=\bar{r}_{i, N-1} e_{i i}(\varepsilon)$ is a $\left(M_{i i}^{-}, M_{i i}^{+}, \bar{r}_{i, N-1} \dot{\delta}_{i i}, \bar{r}_{i, N-1} \dot{G}_{i i}, \bar{r}_{i, N-1} \dot{\varepsilon}_{i i}\right)$-expansion.

The above algorithm can be realized for any sequence $\bar{r}_{i, N-1}=\left\langle r_{i, 1}, \ldots, r_{i, N-1}\right\rangle$, but the invariance of explicit upper bounds for remainders, with respect to permutations $\bar{r}_{i, N}=$ $\left\langle r_{i, 1}, \ldots, r_{i, N-1}, i\right\rangle$ of sequence $\langle 1, \ldots, N\rangle$, can not be guaranteed.

However, the inequality $\bar{r}_{i, N-1} \dot{\delta}_{i i} \geq \delta^{*}$ holds, for any sequence $\bar{r}_{i, N-1}=\left\langle r_{i, 1}, \ldots\right.$, $\left.r_{i, N-1}\right\rangle$, by Theorem 7 .

The algorithm described above can be repeated, for every $i \in \mathbb{X}$.

\section{Asymptotic Expansions with Explicit Upper Bounds for Remainders for Stationary Distributions}

Let us recall the pivotal $\left(n_{i}^{-}, n_{i}^{+}\right)$-expansion for stationary probability $\pi_{i}(\varepsilon)$ of nonlinearly perturbed semi-Markov process $\eta^{(\varepsilon)}(t)$ given, under conditions $\mathbf{A}-\mathbf{E}$, in Theorem 5. This asymptotic expansion has the following form, for $i \in \mathbb{X}$,

$$
\pi_{i}(\varepsilon)=\sum_{l=n_{i}^{-}}^{n_{i}^{+}} c_{i}[l] \varepsilon^{l}+o_{i}\left(\varepsilon^{n_{i}^{+}}\right), \varepsilon \in\left(0, \varepsilon_{0}\right] .
$$

According Theorem 5, the above asymptotic expansion is invariant with respect to the choice of sequence states $\bar{r}_{i, N-1}=\left(r_{i, 1}, \ldots, r_{i, N-1}\right)$ used in the corresponding algorithm, for every $i \in \mathbb{X}$.

The following theorem is the main new result in Part II of this paper. 
Theorem 9 Let conditions $\boldsymbol{A}-\boldsymbol{C}, \boldsymbol{D}^{\prime}$ and $\boldsymbol{E}^{\prime}$ hold for the semi-Markov processes $\eta^{(\varepsilon)}(t)$. Then, for every $i \in \mathbb{X}$, the pivotal $\left(n_{i}^{-}, n_{i}^{+}\right)$-expansion (34) for the stationary probability $\pi_{i}(\varepsilon)$, given in Theorem 5 and obtained as the result of sequential exclusion of states $r_{i, 1}, \ldots, r_{i, N-1}$ from the phase space $\mathbb{X}$ of the processes $\eta^{(\varepsilon)}(t)$, is a $\left(n_{i}^{-}, n_{i}^{+}, \bar{r}_{i, N-1} \delta_{i}, \bar{r}_{i, N-1} G_{i}, \bar{r}_{i, N-1} \varepsilon_{i}\right)$-expansion. Parameters $\bar{r}_{i, N-1} \delta_{i}, \bar{r}_{i, N-1} G_{i}$ and $\bar{r}_{i, N-1} \varepsilon_{i}$ can be computed using the algorithm described below, in the proof of the theorem. Also, inequality $\bar{r}_{i, N-1} \delta_{i}^{*} \geq \delta^{*}$ holds making it possible to rewrite function $\pi_{i}(\varepsilon)$ as the pivotal $\left(n_{i}^{-}, n_{i}^{+}, \delta^{*}, \bar{r}_{i, N-1} G_{i}^{*}, \bar{r}_{i, N-1} \varepsilon_{i}\right)$-expansion, with parameter $\bar{r}_{i, N-1} G_{i}^{*}=\bar{r}_{i, N-1} G_{i}$. $\left(\bar{r}_{i, N-1} \varepsilon_{i}\right)^{\left(\bar{r}_{i, N-1} \delta_{i}-\delta^{*}\right)}$.

Proof Let us choose an arbitrary state $i \in \mathbb{X}$. First, proposition (i) (the multiple summation rule) of Lemmas 3 and 11 should be applied to the pivotal $\left(m_{i}^{-}, m_{i}^{+}\right)$-expansion for the expectation $e_{i}(\varepsilon)=\sum_{j \in \mathbb{Y}_{i}} e_{i j}(\varepsilon)$ given by relation (29), in the proof of Theorem 5. This yields a $\left(m_{i}^{-}, m_{i}^{+}, \dot{\delta}_{i}, \dot{G}_{i}, \dot{\varepsilon}_{i}\right)$-expansion for the expectation $e_{i}(\varepsilon)$, with the corresponding parameters $\dot{\delta}_{i}, \dot{G}_{i}$ and $\dot{\varepsilon}_{i}^{*}$. Second, the propositions (v) (the division rule) of Lemmas 2 and 10 should be applied to the quotient $\pi_{i}(\varepsilon)=\frac{e_{i}(\varepsilon)}{E_{i i}(\varepsilon)}$. The $\left(m_{i}^{-}, m_{i}^{+}, \dot{\delta}_{i}, \dot{G}_{i}, \dot{\varepsilon}_{i}\right)$-expansion for the expectation $e_{i}(\varepsilon)$ and the $\left(M_{i i}^{-}, M_{i i}^{+}, \bar{r}_{i, N-1} \dot{\delta}_{i i}, \bar{r}_{i, N-1} \dot{G}_{i i}, \bar{r}_{i, N-1} \dot{\varepsilon}_{i i}\right)$-expansion for the expectation of hitting time $E_{i i}(\varepsilon)$, given in Theorems 4 and 8 , should be used. This yields the corresponding pivotal $\left(n_{i}^{-}, n_{i}^{+}\right)$-expansion for stationary probability $\pi_{i}(\varepsilon)$, given in Theorem 5 , and proves that this expansion is a $\left(n_{i}^{-}, n_{i}^{+}, \bar{r}_{i, N-1} \delta_{i}, \bar{r}_{i, N-1} G_{i}, \bar{r}_{i, N-1} \varepsilon_{i}\right)$-expansion, with parameters computed in the process of realization of the above algorithm. Inequality $\bar{r}_{i, N-1} \delta_{i} \geq \delta^{*}$ holds, for every sequence $\bar{r}_{i, N-1}$, by proposition (iii) of Lemma 12 .

The explicit upper bounds for remainders in the asymptotic expansions given in Theorem 9 have a clear and informative power-type form. An useful property of these upper bounds is that they are uniform with respect to the perturbation parameter. The recurrent algorithm for finding these upper bounds is computationally effective.

Open Access This article is distributed under the terms of the Creative Commons Attribution 4.0 International License (http://creativecommons.org/licenses/by/4.0/), which permits unrestricted use, distribution, and reproduction in any medium, provided you give appropriate credit to the original author(s) and the source, provide a link to the Creative Commons license, and indicate if changes were made.

\section{Appendix A: Operational Rules for Laurent Asymptotic Expansions}

First of all, we would like to refer to some books, for example, Markushevich (1985) and Hörmander (1990), where one can find basic facts about Laurent series, mainly, connected with representation problems for analytical functions. We, however, are interested in simpler objects and problems that are Laurent asymptotic expansions and operational rules for such expansions, with remainders given in the standard form $o(\cdot)$ or with explicit power type upper bounds. We present the corresponding explicit formulas in eight lemmas and group their proofs in pairs for Lemmas 1 and $9, \ldots, 4$ and 12 . The former lemmas in these pairs give formulas for computing parameters, coefficients and remainders, while the latter lemmas give explicit formulas for computing explicit upper bounds for remainders in the corresponding expansions. Some known or obvious details of the proofs are ommited.

A.1 The formulas given in Lemmas 1 and 9 are quite obvious. 
A.2 The same relates to formulas in propositions (i) (the multiplication by a constant rule) of Lemmas 2 and 10.

Proposition (ii) (the summation rules) of Lemmas 2 and 10 can be obtained by simple accumulation of coefficients for different powers of $\varepsilon$ and terms accumulated in the corresponding remainders, and, then, by using obvious upper bounds for absolute values of sums of terms accumulated in the corresponding remainders.

Proposition (iii) (the multiplication rule) of Lemma 2 can be proved by multiplication of the corresponding asymptotic expansions $A(\varepsilon)$ and $B(\varepsilon)$ and accumulation of coefficients for powers $\varepsilon^{l}$ for $l=h_{C}, \ldots, k_{C}$ in their product,

$$
\begin{aligned}
C(\varepsilon)= & A(\varepsilon) B(\varepsilon)=\left(a_{h_{A}} \varepsilon^{h_{A}}+\cdots+a_{k_{A}} \varepsilon^{k_{A}}+o_{A}\left(\varepsilon^{k_{A}}\right)\right) \\
& \times\left(b_{h_{B}} \varepsilon^{h_{B}}+\cdots+b_{k_{B}} \varepsilon^{k_{B}}+o_{B}\left(\varepsilon^{k_{B}}\right)\right) \\
= & \sum_{h_{C} \leq l \leq k_{C}} \sum_{i+j=l, h_{A} \leq i \leq k_{A}, h_{B} \leq j \leq k_{B}} a_{i} b^{l} \\
& +\sum_{k_{C}<i+j, h_{A} \leq i \leq k_{A}, h_{B} \leq j \leq k_{B}} a_{i} b_{j} \varepsilon^{i+j}+\sum_{h_{B} \leq j \leq k_{B}} b_{j} \varepsilon^{j} o_{A}\left(\varepsilon^{k_{A}}\right) \\
& +\sum_{h_{A} \leq i \leq k_{A}} a_{i} \varepsilon^{i} o_{B}\left(\varepsilon^{k_{B}}\right)+o_{A}\left(\varepsilon^{k_{A}}\right) o_{B}\left(\varepsilon^{k_{B}}\right) \\
= & \sum_{h_{C} \leq l \leq k_{C}} c_{l} \varepsilon^{l}+o_{C}\left(\varepsilon^{k_{C}}\right),
\end{aligned}
$$

where

$$
\begin{aligned}
o_{C}\left(\varepsilon^{k_{C}}\right)= & \sum_{k_{C}<i+j, h_{A} \leq i \leq k_{A}, h_{B} \leq j \leq k_{B}} a_{i} b_{j} \varepsilon^{i+j}+\sum_{h_{B} \leq j \leq k_{B}} b_{j} \varepsilon^{j} o_{A}\left(\varepsilon^{k_{A}}\right) \\
& +\sum_{h_{A} \leq i \leq k_{A}} a_{i} \varepsilon^{i} o_{B}\left(\varepsilon^{k_{B}}\right)+o_{A}\left(\varepsilon^{k_{A}}\right) o_{B}\left(\varepsilon^{k_{B}}\right) .
\end{aligned}
$$

Obviously, $\frac{o_{C}\left(\varepsilon^{k} C\right)}{\varepsilon^{k} C} \rightarrow 0$ as $\varepsilon \rightarrow 0$. It should be noted that the accumulation of coefficients for powers $\varepsilon^{l}$ can be made in Eq. 35 only up to the maximal value $l=k_{C}=$ $\left(k_{A}+h_{B}\right) \wedge\left(k_{B}+h_{A}\right)$, because of the presence in the expression for remainder $o_{C}\left(\varepsilon^{k_{C}}\right)$ terms $b_{h_{B}} \varepsilon^{h_{B}} o_{A}\left(\varepsilon^{k_{A}}\right)$ and $a_{h_{A}} \varepsilon^{h_{A}} o_{B}\left(\varepsilon^{k_{B}}\right)$.

Also, relation (36) readily implies relations (a)-(c), which determines parameters $\delta_{C}, G_{C}, \varepsilon_{C}$ and $\tilde{\delta}_{C}, \tilde{G}_{C}, \tilde{\varepsilon}_{C}$ in proposition (iii) of Lemma 10 , in particular, the following inequalities take place, for $\varepsilon \in\left(0, \varepsilon_{C}\right]$,

$$
\begin{aligned}
\left|\varepsilon^{-k_{C}-\delta_{C}} o_{C}\left(\varepsilon^{k_{C}}\right)\right| \leq & \sum_{k_{C}<i+j, h_{A} \leq i \leq k_{A}, h_{B} \leq j \leq k_{B}}\left|a_{i}\right|\left|b_{j}\right| \varepsilon_{C}^{i+j-k_{C}-\delta_{C}} \\
& +G_{A} \sum_{h_{B} \leq j \leq k_{B}}\left|b_{j}\right| \varepsilon_{C}^{j+k_{A}+\delta_{A}-k_{C}-\delta_{C}} \\
& +G_{B} \sum_{h_{A} \leq i \leq k_{A}}\left|a_{i}\right| \varepsilon_{C}^{i+k_{B}+\delta_{B}-k_{C}-\delta_{C}}+G_{A} G_{B} \varepsilon_{C}^{k_{A}+k_{B}+\delta_{A}+\delta_{B}-k_{C}-\delta_{C}} \\
\leq & G_{C}=F_{A} F_{B}\left(k_{A}-h_{A}+1\right)\left(k_{B}-h_{B}+1\right) \\
& +G_{A} F_{B}\left(k_{B}-h_{B}+1\right)+G_{B} F_{A}\left(k_{A}-h_{A}+1\right)+G_{A} G_{B} \\
= & \left(F_{A}\left(k_{A}-h_{A}+1\right)+G_{A}\right)\left(F_{B}\left(k_{B}-h_{B}+1\right)+G_{B}\right) .
\end{aligned}
$$


Parameter $\varepsilon_{C} \in(0,1]$ is taken to nonnegative powers in all terms appearing in the sums on the right hand side of the first inequality in relation (37). This makes it possible to estimate these sums from above by the corresponding simpler expressions replacing parameter $\varepsilon_{C}$ by 1 and absolute values of coefficients $a_{i}$ and $b_{j}$ by their maximum values.

Analogous approach is used below.

The assumptions of proposition (iv) in Lemma 2 imply that $\varepsilon^{-h_{B}} B(\varepsilon) \rightarrow b_{h_{B}} \neq 0$ as $\varepsilon \rightarrow 0$. This relation implies that there exists $0<\varepsilon_{0}^{\prime} \leq \varepsilon_{0}$ such that $B(\varepsilon) \neq 0$ for $\varepsilon \in\left(0, \varepsilon_{0}^{\prime}\right]$, and, thus, function $C(\varepsilon)=\frac{1}{B(\varepsilon)}$ is well defined for $\varepsilon \in\left(0, \varepsilon_{0}^{\prime}\right]$.

Note that $h_{B} \leq k_{B}$. The assumptions of proposition (iv) of Lemma 2 imply that, $\varepsilon^{h_{B}} C(\varepsilon)=\left(b_{h_{B}}+\cdots+b_{k_{B}} \varepsilon^{k_{B}-h_{B}}+o_{B}\left(\varepsilon^{h_{B}}\right) \varepsilon^{-h_{B}}\right)^{-1} \rightarrow b_{h_{B}}^{-1}=c_{h_{C}}$ as $\varepsilon \rightarrow 0$. This relation means that function $\varepsilon^{h_{B}} C(\varepsilon)$ can be represented in the form $\varepsilon^{h_{B}} C(\varepsilon)=c_{h_{C}}+o(1)$, where $c_{h_{C}}=b_{h_{B}}^{-1}$, or, equivalently, that the following representation takes place, $C(\varepsilon)=$ $c_{h_{C}} \varepsilon^{-h_{B}}+o_{1}\left(\varepsilon^{-h_{B}}\right), \varepsilon \in\left(0, \varepsilon_{0}^{\prime}\right]$, where $\frac{o_{1}\left(\varepsilon^{-h_{B}}\right)}{\varepsilon^{-h_{B}}} \rightarrow 0$ as $\varepsilon \rightarrow 0$.

The latter two relations prove proposition (iv) of Lemma 2, for the case $h_{B}=k_{B}$. Indeed, these relations mean that function $C(\varepsilon)=\frac{1}{B(\varepsilon)}$ can be represented in the form of $\left(h_{C}, k_{C}\right)$ expansion with parameters $h_{C}=-h_{B}, k_{C}=k_{B}-2 h_{B}=-h_{B}=h_{C}$ and coefficient $c_{h_{C}}=$ $b_{h_{B}}^{-1}$. Moreover, since $B(\varepsilon) \cdot C(\varepsilon) \equiv 1,0<\varepsilon \leq \varepsilon_{0}^{\prime}$, remainder $c_{1}(\varepsilon)$ can be found from the following relation, $\left(b_{h_{B}} \varepsilon^{h_{B}}+o\left(\varepsilon^{h_{B}}\right)\right)\left(c_{h_{C}} \varepsilon^{-h_{B}}+o_{1}\left(\varepsilon^{-h_{B}}\right)\right) \equiv 1$ that yields formula,

$$
o_{1}\left(\varepsilon^{-h_{B}}\right)=-\frac{c_{h_{C}} \varepsilon^{-h_{B}} o_{B}\left(\varepsilon^{h_{B}}\right)}{b_{h_{B}} \varepsilon^{h_{B}}+o_{B}\left(\varepsilon^{h_{B}}\right)} .
$$

This is formula (c) from proposition (iv) of Lemma 2, for the case $h_{B}=k_{B}$. Note that, in the case $h_{B}=k_{B}$, the above asymptotic expansion for function $C(\varepsilon)$ can not be extended. Indeed, $\varepsilon^{h_{B}-1} o_{1}\left(\varepsilon^{-h_{B}}\right)=\varepsilon^{h_{B}-1}\left(C(\varepsilon)-c_{h_{C}} \varepsilon^{-h_{B}}\right)=-\frac{c_{h_{C}}}{b_{h_{B}}+o_{B}\left(\varepsilon^{h_{B}}\right) \varepsilon^{-h_{B}}} \frac{o_{B}\left(\varepsilon^{h_{B}}\right) \varepsilon^{-h_{B}}}{\varepsilon}$. The term $\frac{o_{B}\left(\varepsilon^{h} B\right) \varepsilon^{-h_{B}}}{\varepsilon}$ on the right hand side in the latter relation has an uncertain asymptotic behavior as $\varepsilon \rightarrow 0$.

Let us now assume that $h_{B}+1 \leq k_{B}$. In this case, the assumptions of proposition (iv) of Lemma 2 and the above asymptotic relations imply that $\varepsilon^{h_{B}-1} o_{1}\left(\varepsilon^{-h_{B}}\right)=$ $\varepsilon^{h_{B}-1}\left(C(\varepsilon)-c_{h_{C}} \varepsilon^{-h_{B}}\right)=\left(b_{h_{B}}+\cdots+b_{k_{B}} \varepsilon^{k_{B}-h_{B}}+o_{B}\left(\varepsilon^{k_{B}}\right) \varepsilon^{-h_{B}}\right)^{-1}\left(-b_{h_{B}+1} c_{h_{C}}-\cdots\right.$ $\left.-b_{k_{B}} c_{h_{C}} \varepsilon^{k_{B}-h_{B}-1}-o_{B}\left(\varepsilon^{k_{B}}\right) c_{h_{C}} \varepsilon^{-h_{B}-1}\right) \rightarrow-b_{h_{B}}^{-1} b_{h_{B}+1} c_{h_{C}}=c_{h_{C}+1}$ as $\varepsilon \rightarrow 0$. This relation means that function $\varepsilon^{h_{B}-1} \times o_{1}\left(\varepsilon^{-h_{B}}\right)$ can be represented in the form $\varepsilon^{h_{B}-1} o_{1}\left(\varepsilon^{-h_{B}}\right)=$ $c_{h_{C}+1}+o(1)$, where $c_{h_{C}+1}=-b_{h_{B}}^{-1} b_{h_{B}+1} c_{h_{C}}$, or, equivalently, that the following representation takes place, $C(\varepsilon)=c_{h_{C}} \varepsilon^{-h_{B}}+c_{h_{C}+1} \varepsilon^{-h_{B}+1}+o_{2}\left(\varepsilon^{-h_{B}+1}\right), \varepsilon \in\left(0, \varepsilon_{0}^{\prime}\right]$, where $\frac{o_{2}\left(\varepsilon^{-h_{B}+1}\right)}{\varepsilon^{-h_{B}+1}} \rightarrow 0$ as $\varepsilon \rightarrow 0$.

$\varepsilon^{-}$The latter two relations prove proposition (iv) of Lemmas 2, for the case $h_{B}+1=k_{B}$. Indeed, these relations mean that function $C(\varepsilon)$ can be represented in the form of $\left(h_{C}, k_{C}\right)$ expansion with parameters $h_{C}=-h_{B}, k_{C}=k_{B}-2 h_{B}=-h_{B}+1=h_{C}+1$ and coefficients $c_{h_{C}}=b_{h_{B}}^{-1}, c_{h_{C}+1}=-b_{h_{B}}^{-1} b_{h_{B}+1} c_{h_{C}}$. Moreover, since $B(\varepsilon) \cdot C(\varepsilon) \equiv 1$, the remainder $o_{2}\left(\varepsilon^{-h_{B}+1}\right)$ can be found from the following relation, $\left(b_{h_{B}} \varepsilon^{h_{B}}+b_{h_{B}+1} \varepsilon^{h_{B}+1}+\right.$ $\left.o\left(\varepsilon^{h_{B}+1}\right)\right) \times\left(c_{h_{C}} \varepsilon^{-h_{B}}+c_{h_{C}+1} \varepsilon^{-h_{B}+1}+o_{2}\left(\varepsilon^{-h_{B}+1}\right)\right) \equiv 1$. This relation yields formula,

$$
o_{2}\left(\varepsilon^{-h_{B}+1}\right)=-\frac{b_{h_{B}+1} c_{h_{C}+1} \varepsilon^{2}+\left(c_{h_{C}} \varepsilon^{-h_{B}}+c_{h_{C}+1} \varepsilon^{-h_{B}+1}\right) o_{B}\left(\varepsilon^{h_{B}+1}\right)}{b_{h_{B}} \varepsilon^{h_{B}}+b_{h_{B}+1} \varepsilon^{h_{B}+1}+o_{B}\left(\varepsilon^{h_{B}+1}\right)} .
$$


This is formula (c) from proposition (iv) of Lemma 2, for the case $h_{B}+1=k_{B}$. Note that, in the case $h_{B}+1=k_{B}$, the above asymptotic expansion for function $C(\varepsilon)$ can not be extended. Indeed, $\varepsilon^{h_{B}-2} o_{2}(\varepsilon)=\varepsilon^{h_{B}-2}\left(C(\varepsilon)-c_{h_{C}} \varepsilon^{-h_{B}}-c_{h_{C}+1} \varepsilon^{-h_{B}+1}\right)=$ $-\frac{b_{h_{B}+1} c_{h_{C}+1}+c_{h_{C}+1} o_{B}\left(\varepsilon^{h_{B}+1}\right) \varepsilon^{-h_{B}-1}}{b_{h_{B}}+b_{h_{B}+1} \varepsilon+o_{B}\left(\varepsilon^{h_{B}+1}\right) \varepsilon^{-h_{B}}}-\frac{c_{h_{C}}}{b_{h_{B}}+b_{h_{B}+1} \varepsilon+o_{B}\left(\varepsilon^{h_{B}+1}\right) \varepsilon^{-h_{B}}} \frac{o_{B}\left(\varepsilon^{h_{B}+1}\right) \varepsilon^{-h_{B}-1}}{\varepsilon}$. The term $\frac{o_{B}\left(\varepsilon^{h}{ }^{+1}\right) \varepsilon^{-h_{B}-1}}{\varepsilon}$ on the right hand side in the latter relation has an uncertain asymptotic behavior as $\varepsilon \rightarrow 0$.

We can repeat the above arguments for the general case $h_{B}+n=k_{B}$, for any $n=0,1, \ldots$ and to prove that, in the case $h_{B}+n=k_{B}$, function $C(\varepsilon)$ can be represented in the form of $\left(h_{C}, k_{C}\right.$ )-expansion with parameters $h_{C}=-h_{B}, k_{C}=k_{B}-2 h_{B}=-h_{B}+n=h_{C}+n$ and coefficients $c_{h_{C}}, \ldots, c_{k_{C}}$ given in proposition (iv) of Lemma 2. Moreover, identity $B(\varepsilon)$. $C(\varepsilon) \equiv 1,0<\varepsilon \leq \varepsilon_{0}^{\prime}$, let us find the corresponding remainder $o_{C}\left(\varepsilon^{k_{C}}\right)$ from the following relation,

$$
\left(b_{h_{B}} \varepsilon^{h_{B}}+\cdots+b_{k_{B}} \varepsilon^{k_{B}}+o_{B}\left(\varepsilon^{k_{B}}\right)\right) \times\left(c_{h_{C}} \varepsilon^{h_{C}}+\cdots+c_{h_{C}} \varepsilon^{k_{C}}+o_{C}\left(\varepsilon^{k_{C}}\right)\right) \equiv 1 .
$$

Proposition (iii) of Lemma 2, applied to the product on the left hand side in relation (40), permits to represent this product in the form of $(h, k)$-expansion with parameters $h=$ $h_{B}+h_{C}=h_{B}-h_{B}=0$ and $k=\left(k_{B}+h_{C}\right) \wedge\left(h_{B}+k_{C}\right)=\left(k_{B}-h_{B}\right) \wedge\left(k_{B}-2 h_{B}+h_{B}\right)=$ $k_{B}-h_{B}$. By canceling coefficient for $\varepsilon^{l}$ on the left and right hand sides in relation (40), for $l=0, \ldots, k_{B}-h_{B}$, and then, by solving Eq. 40 with respect to the remainder $o_{C}\left(\varepsilon^{k_{C}}\right)$, we get the formula for this remainder given in proposition (iv) of Lemma 2,

$$
\begin{aligned}
o_{C}\left(\varepsilon^{k_{C}}\right)= & -\frac{\sum_{k_{B}-h_{B}<i+j, h_{B} \leq i \leq k_{B}, h_{C} \leq j \leq k_{C}} b_{i} c_{j} \varepsilon^{i+j}+\sum_{h_{C} \leq j \leq k_{C}} c_{j} \varepsilon^{j} o_{B}\left(\varepsilon^{k_{B}}\right)}{b_{h_{B}} \varepsilon^{h_{B}}+\cdots+b_{k_{B}} \varepsilon^{k_{B}}+o_{B}\left(\varepsilon^{k_{B}}\right)} \\
= & -\frac{\sum_{k_{B}-h_{B}<i+j, h_{B} \leq i \leq k_{B}, h_{C} \leq j \leq k_{C}} b_{i} c_{j} \varepsilon^{i+j-h_{B}}}{b_{h_{B}}+\cdots+b_{k_{B}} \varepsilon^{k_{B}-h_{B}}+o_{B}\left(\varepsilon^{k_{B}}\right) \varepsilon^{h_{B}}} \\
& -\frac{\sum_{h_{C} \leq j \leq k_{C}} c_{j} \varepsilon^{j-h_{B}} o_{B}\left(\varepsilon^{k_{B}}\right)}{b_{h_{B}}+\cdots+b_{k_{B}} \varepsilon^{k_{B}-h_{B}}+o_{B}\left(\varepsilon^{k_{B}}\right) \varepsilon^{-h_{B}}} .
\end{aligned}
$$

The assumptions made in proposition (iv) of Lemma 2, imply that $B(\varepsilon) \neq 0$ and the following inequality holds for $0<\varepsilon \leq \varepsilon_{C}$, where $\varepsilon_{C}$ is given in proposition (iv) of Lemma 2,

$$
\begin{aligned}
\mid b_{h_{B}} & +b_{h_{B}+1} \varepsilon+\cdots+b_{k_{B}} \varepsilon^{k_{B}-h_{B}}+o_{B}\left(\varepsilon^{k_{B}}\right) \varepsilon^{-h_{B}} \mid \\
& \geq\left|b_{h_{B}}\right|-\left(\left|b_{h_{B}+1}\right| \varepsilon+\cdots+\left|b_{k_{B}}\right| \varepsilon^{k_{B}-h_{B}}+G_{B} \varepsilon^{k_{B}-h_{B}+\delta_{B}}\right) \\
& \geq \frac{\left|b_{h_{B}}\right|}{2}>0 .
\end{aligned}
$$

The existence of $\varepsilon_{0}^{\prime}$ declared in proposition (iv) of Lemma 2 is obvious. For example, one can choose $\varepsilon_{0}^{\prime}=\varepsilon_{C}$. It is also useful to note that formulas given in proposition (iv) of Lemma 2 imply that $\varepsilon_{C}=\varepsilon_{B} \wedge \tilde{\varepsilon}_{B} \in\left(0, \varepsilon_{0}\right]$, since $\varepsilon_{B} \in\left(0, \varepsilon_{0}\right]$ and $\tilde{\varepsilon}_{B} \in(0, \infty)$. 
The assumptions made in proposition (iv) of Lemma 2 and inequality (42) imply that the following inequality holds, for $0<\varepsilon \leq \varepsilon_{C}$,

$$
\begin{aligned}
\left|\varepsilon^{-k_{C}-\delta_{C}} o_{C}\left(\varepsilon^{k_{C}}\right)\right|= & \left|\varepsilon^{-k_{B}+2 h_{B}-\delta_{B}} o_{C}\left(\varepsilon^{k_{C}}\right)\right| \\
\leq & \left(\frac{\left|b_{h_{B}}\right|}{2}\right)^{-1}\left(G_{B} \sum_{h_{C} \leq j \leq k_{C}}\left|c_{j}\right| \varepsilon_{C}^{j+h_{B}}\right. \\
& \left.+\sum_{k_{B}-h_{B}<i+j, h_{B} \leq i \leq k_{B}, h_{C} \leq j \leq k_{C}}\left|b_{i}\right|\left|c_{j}\right| \varepsilon_{C}^{i+j-k_{B}+h_{B}-\delta_{B}}\right) \\
\leq & G_{C}=\left(\frac{\left|b_{h_{B}}\right|}{2}\right)^{-1}\left(G_{B} F_{C}\left(k_{C}-h_{C}+1\right)\right. \\
& \left.+F_{B} F_{C}\left(k_{B}-h_{B}+1\right)\left(k_{C}-h_{C}+1\right)\right) \\
= & \left(\frac{\left|b_{h_{B}}\right|}{2}\right)^{-1}\left(G_{B}+F_{B}\left(k_{B}-h_{B}+1\right)\right) F_{C}\left(k_{C}-h_{C}+1\right) .
\end{aligned}
$$

Inequality (43) proofs proposition (iv) of Lemma 10.

Propositions (v) of Lemmas 2 and 10 and relations (a)-(c) given in these propositions can be obtained by direct application, respectively, of propositions (iii) and (iv) of Lemmas 2 and 10 to the product $D(\varepsilon)=A(\varepsilon) \cdot \frac{1}{B(\varepsilon)}$. This yields that $D(\varepsilon)$ is is a $\left(h_{D}, k_{D}\right)$-expansion, with parameters $h_{D}=h_{A}-h_{B}$ and $k_{D}=\left(k_{A}-h_{B}\right) \wedge\left(k_{B}-2 h_{B}+h_{A}\right)$. Now, when it is already known that $D(\varepsilon)$ is a $\left(h_{D}, k_{D}\right)$-expansion, multiplication of $D(\varepsilon)$ by $B(\varepsilon)$ yields the following relation holding for $\varepsilon \in\left(0, \varepsilon_{0}^{\prime}\right]$,

$$
\begin{aligned}
A(\varepsilon)= & D(\varepsilon) B(\varepsilon)=a_{h_{A}} \varepsilon^{h_{A}}+\cdots+a_{h_{A}} \varepsilon^{k_{A}}+o_{A}\left(\varepsilon^{k_{A}}\right) \\
= & \left(d_{h_{D}} \varepsilon^{h_{D}}+\cdots+d_{h_{D}} \varepsilon^{k_{D}}+o_{D}\left(\varepsilon^{k_{D}}\right)\right) \\
& \times\left(b_{h_{B}} \varepsilon^{h_{B}}+\cdots+b_{h_{B}} \varepsilon^{k_{B}}+o_{B}\left(\varepsilon^{k_{B}}\right)\right) .
\end{aligned}
$$

By equating coefficients for powers $\varepsilon^{l}$ for $l=h_{D}, \ldots, k_{D}$ on the left and right hand sides of the third equality in relation (44), we get formulas (b) for coefficients $d_{h_{d}}, \ldots, d_{k_{D}}$ given in proposition (v) of Lemma 2.

Proposition (iii) of Lemma 2, applied to the product on the right hand side in Eq. 44, permits to represent this product in the form of $(h, k)$-expansion with parameters $h=h_{B}+$ $h_{D}=h_{B}+h_{A}-h_{B}=h_{A}$ and $k=\left(k_{D}+h_{B}\right) \wedge\left(k_{B}+h_{D}\right)=\left(\left(k_{A}-h_{B}\right) \wedge\left(k_{B}-2 h_{B}+\right.\right.$ $\left.\left.h_{A}\right)+h_{B}\right) \wedge\left(k_{B}+h_{A}-h_{B}\right)=k_{A} \wedge\left(k_{B}+h_{A}-h_{B}\right)$. By canceling coefficient for $\varepsilon^{l}$ on the left and right hand sides in relation (44), for $l=h_{A}, \ldots, k_{A} \wedge\left(k_{B}+h_{A}-h_{B}\right)$, and 
then, by solving Eq. 44 with respect to the remainder $o_{D}\left(\varepsilon^{k_{D}}\right)$, we get the formula (c) for this remainder given in proposition (v) of Lemma 2,

$$
\begin{aligned}
& o_{D}\left(\varepsilon^{k_{D}}\right)=\frac{\sum_{k_{A} \wedge\left(k_{B}+h_{A}-h_{B}\right)<l \leq k_{A}} a_{l} \varepsilon^{l}+o_{A}\left(\varepsilon^{k_{A}}\right)}{b_{h_{B}} \varepsilon^{h_{B}}+\cdots+b_{k_{B}} \varepsilon^{k_{B}}+o_{B}\left(\varepsilon^{k_{B}}\right)} \\
& -\frac{\sum_{k_{A} \wedge\left(k_{B}+h_{A}-h_{B}\right)<i+j, h_{B} \leq i \leq k_{B}, h_{D} \leq j \leq k_{D}} b_{i} d_{j} \varepsilon^{i+j}}{b_{h_{B}} \varepsilon^{h_{B}}+\cdots+b_{k_{B}} \varepsilon^{k_{B}}+o_{B}\left(\varepsilon^{k_{B}}\right)} \\
& -\frac{\sum_{h_{D} \leq j \leq k_{D}} d_{j} \varepsilon^{j} o_{B}\left(\varepsilon^{k_{B}}\right)}{b_{h_{B}} \varepsilon^{h_{B}}+\cdots+b_{k_{B}} \varepsilon^{k_{B}}+o_{B}\left(\varepsilon^{k_{B}}\right)} \\
& =\frac{\sum_{k_{A} \wedge\left(k_{B}+h_{A}-h_{B}\right)<l \leq k_{A}} a_{l} \varepsilon^{l-h_{B}}+o_{A}\left(\varepsilon^{k_{A}}\right) \varepsilon^{-h_{B}}}{b_{h_{B}}+\cdots+b_{k_{B}} \varepsilon^{k_{B}-h_{B}}+o_{B}\left(\varepsilon^{k_{B}}\right) \varepsilon^{-h_{B}}} \\
& -\frac{\sum_{k_{A} \wedge\left(k_{B}+h_{A}-h_{B}\right)<i+j, h_{B} \leq i \leq k_{B}, h_{D} \leq j \leq k_{D}} b_{i} d_{j} \varepsilon^{i+j-h_{B}}}{b_{h_{B}}+\cdots+b_{k_{B}} \varepsilon^{k_{B}-h_{B}}+o_{B}\left(\varepsilon^{k_{B}}\right) \varepsilon^{-h_{B}}} \\
& -\frac{\sum_{h_{D} \leq j \leq k_{D}} d_{j} \varepsilon^{j-h_{B}} o_{B}\left(\varepsilon^{k_{B}}\right)}{b_{h_{B}}+\cdots+b_{k_{B}} \varepsilon^{k_{B}-h_{B}}+o_{B}\left(\varepsilon^{k_{B}}\right) \varepsilon^{-h_{B}}} .
\end{aligned}
$$

Inequality (42), the assumptions made in proposition (v) of Lemma 10 and relation (45) finally imply that the following inequality holds, for $0<\varepsilon \leq \varepsilon_{D}$ given in relation (c) of this proposition,

$$
\begin{aligned}
\left|\varepsilon^{-k_{D}-\delta_{D}} o_{D}\left(\varepsilon^{k_{D}}\right)\right| \leq & \left(\frac{\left|b_{h_{B}}\right|}{2}\right)^{-1} \\
& \times\left(\sum_{k_{A} \wedge\left(h_{A}+k_{B}-h_{B}\right)<i \leq k_{A}}\left|a_{i}\right| \varepsilon_{D}^{i-h_{B}-k_{D}-\delta_{D}}+G_{A} \varepsilon_{D}^{k_{A}+\delta_{A}-h_{B}-k_{D}-\delta_{D}}\right. \\
& +\sum_{k_{A} \wedge\left(h_{A}+k_{B}-h_{B}\right)<i+j, h_{B} \leq i \leq k_{B}, h_{D} \leq j \leq k_{D}}\left|b_{i}\right|\left|d_{j}\right| \varepsilon_{D}^{i+j-k_{D}-h_{B}-\delta_{D}} \\
& \left.+G_{B} \sum_{h_{D} \leq j \leq k_{D}}\left|d_{j}\right| \varepsilon_{D}^{j+k_{B}+\delta_{B}-h_{B}-k_{D}-\delta_{D}}\right) \\
\leq & G_{D}=\left(\frac{\left|b_{h_{B}}\right|}{2}\right)^{-1}\left(F_{A}\left(k_{A}-k_{A} \wedge\left(h_{A}+k_{B}-h_{B}\right)\right)+G_{A}\right. \\
& \left.+F_{B} F_{D}\left(k_{B}-h_{B}+1\right)\left(k_{D}-h_{D}+1\right)+G_{B} F_{D}\left(k_{D}-h_{D}+1\right)\right) .
\end{aligned}
$$

Inequality (46) yields relations (a)-(c) given in proposition (v) of Lemma 10.

A.3 Lemma 3 is a direct corollary of Lemma 2. Proofs of propositions (i) and (ii) in Lemma 11 are analogous to proofs of the corresponding propositions in Lemma 10. Proposition (iii) of Lemma 11 is obvious.

A.4 The first two identities for Laurent asymptotic expansions given in proposition (i) of Lemma 4 are obvious. The third identity given in this proposition follows in an obvious 
way from proposition (i) of Lemma 2. By applying propositions (iii) and (iv) of Lemma 2 to the product $C(\varepsilon)=A(\varepsilon) \cdot A(\varepsilon)^{-1}$, we get parameters $h_{C}=h_{A \cdot A^{-1}}=h_{A}-h_{A}=$ $0, k_{C}=k_{A \cdot A^{-1}}=\left(k_{A}-h_{A}\right) \wedge\left(k_{A}-2 h_{A}+h_{A}\right)=k_{A}-h_{A}$ and coefficients $c_{n}=$ $\mathrm{I}(n=0), n=0, \ldots, k_{C}$. Also, relations (40) and (41) imply that the elimination identity $A(\varepsilon) \cdot A(\varepsilon)^{-1} \equiv 1$ holds, since the remainder of Laurent asymptotic expansion for function $A(\varepsilon)^{-1}$ is given by formula (c) from proposition (iv) of Lemma 2.

Propositions (ii) and (iii) of Lemma 4 in the parts concerned commutative property of summation and multiplication operations follow from, respectively, propositions (ii) and (iii) of Lemma 2.

Let $D(\varepsilon)=(A(\varepsilon)+B(\varepsilon))+C(\varepsilon)=A(\varepsilon)+(B(\varepsilon)+C(\varepsilon))$. Using propositions (ii) of Lemma 2, we get, $h_{D}=h_{(A+B)+C}=\left(h_{A} \wedge h_{B}\right) \wedge h_{C}=h_{A} \wedge\left(h_{B} \wedge h_{C}\right)=h_{A+(B+C)}$ and $k_{D}=k_{(A+B)+C}=\left(k_{A} \wedge k_{B}\right) \wedge k_{C}=k_{A} \wedge\left(k_{B} \wedge k_{C}\right)=k_{A+(B+C)}$. These relations and Lemma 1 imply equalities for the corresponding coefficients and remainders, for the asymptotic expansions of functions $(A(\varepsilon)+B(\varepsilon))+C(\varepsilon)$ and $A(\varepsilon)+(B(\varepsilon)+C(\varepsilon))$. The above remarks prove proposition (ii) of Lemma 4 in the part concerned with the associative property of summation operation for Laurent asymptotic expansions.

Let $D(\varepsilon)=(A(\varepsilon) \cdot B(\varepsilon)) \cdot C(\varepsilon)=A(\varepsilon) \cdot(B(\varepsilon) \cdot C(\varepsilon))$. Using propositions (iii) of Lemma 2, we get, $h_{D}=h_{(A \cdot B) \cdot C}=h_{A \cdot B}+h_{C}=h_{A}+h_{B}+h_{C}=h_{A}+h_{B \cdot C}=h_{A \cdot(B \cdot C)}$ and $\left.k_{D}=k_{(A \cdot B) \cdot C}=\left(k_{A \cdot B}+h_{C}\right) \wedge\left(k_{C}+h_{A \cdot B}\right)=\left(\left(k_{A}+h_{B}\right) \wedge\left(k_{B}+h_{A}\right)\right)+h_{C}\right) \wedge$ $\left(k_{C}+\left(h_{A}+h_{B}\right)\right)=\left(k_{A}+h_{B}+h_{C}\right) \wedge\left(k_{B}+h_{A}+h_{C}\right) \wedge\left(k_{C}+h_{A}+h_{B}\right)=\left(k_{A}+\left(h_{B}+\right.\right.$ $\left.\left.\left.h_{C}\right)\right) \wedge\left(\left(k_{B}+h_{C}\right) \wedge\left(k_{C}+h_{B}\right)\right)+h_{A}\right)=\left(k_{A}+h_{B \cdot C}\right) \wedge\left(k_{B \cdot C}+h_{A}\right)=k_{A \cdot(B \cdot C)}$. These relations and Lemma 1 imply equalities for the corresponding coefficients and remainders, for the asymptotic expansions of functions $(A(\varepsilon) \cdot B(\varepsilon)) \cdot C(\varepsilon)$ and $A(\varepsilon) \cdot(B(\varepsilon) \cdot C(\varepsilon))$. The above remarks prove proposition (iii) of Lemma 4 in the part concerned with the associative property of multiplication operation for Laurent asymptotic expansions.

Let $D(\varepsilon)=(A(\varepsilon)+B(\varepsilon)) \cdot C(\varepsilon)=A(\varepsilon) \cdot C(\varepsilon)+B(\varepsilon) \cdot C(\varepsilon)$. Using propositions (ii) and (iii) of Lemma 2, we get, $h_{D}=h_{(A+B) \cdot C}=h_{A+B}+h_{C}=h_{A} \wedge h_{B}+h_{C}=\left(h_{A}+h_{C}\right) \wedge$ $\left(h_{B}+h_{C}\right)=h_{A \cdot C} \wedge h_{B \cdot C}=h_{A \cdot C+B \cdot C}$ and $k_{D}=k_{(A+B) \cdot C}=\left(k_{A+B}+h_{C}\right) \wedge\left(k_{C}+h_{A+B}\right)=$ $\left(k_{A} \wedge k_{B}+h_{C}\right) \wedge\left(k_{C}+h_{A} \wedge h_{B}\right)=\left(k_{A}+h_{C}\right) \wedge\left(k_{B}+h_{C}\right) \wedge\left(k_{C}+h_{A}\right) \wedge\left(k_{C}+h_{B}\right)=$ $\left(\left(k_{A}+h_{C}\right) \wedge\left(k_{C}+h_{A}\right)\right) \wedge\left(\left(k_{B}+h_{C}\right) \wedge\left(k_{C}+h_{B}\right)\right)=k_{A \cdot C} \wedge k_{B \cdot C}=k_{A \cdot C+B \cdot C}$. These relations and Lemma 1 imply equalities for the corresponding coefficients and remainders, for the asymptotic expansions of functions $(A(\varepsilon)+B(\varepsilon)) \cdot C(\varepsilon)$ and $A(\varepsilon) \cdot C(\varepsilon)+B(\varepsilon) \cdot C(\varepsilon)$. The above remarks prove proposition (iv) of Lemma 4 concerned with the distributive property of summation and multiplication operations for Laurent asymptotic expansions.

Propositions (i) and (ii) of Lemma 12 readily follow from, respectively, propositions (ii) and (iii) of Lemma 2. Finally, proposition (iii) of Lemma 12 follows from relations $\delta_{c A}=\delta_{A}, \delta_{A+B}, \delta_{A \cdot B}, \delta_{A / B} \geq \delta_{A} \wedge \delta_{B}$ and $\delta_{A_{1}+\cdots+A_{N}}, \delta_{A_{1} \times \cdots \times A_{N}} \geq \min _{1 \leq m \leq N} \delta_{A_{m}}$, given, respectively, in Lemmas 10 and 11 .

\section{Appendix B: An Example}

In conclusion, let us consider a numerical example.

We assume that the semi-Markov process $\eta^{(\varepsilon)}(t)$ is, for every $\varepsilon \in\left(0, \varepsilon_{0}\right]$, a semi-Markov process with the phase space $\mathbb{X}=\{1,2,3\}$.

The transition sets are $\mathbb{Y}_{1}=\{1,2\}, \mathbb{Y}_{2}=\{1,2,3\}, \mathbb{Y}_{3}=\{1,2\}$. 
The $3 \times 3$ matrix of transition probabilities $\left\|p_{i j}(\varepsilon)\right\|$, for the corresponding embedded Markov chain $\eta_{n}^{(\varepsilon)}$, has the following form,

$$
\left\|\begin{array}{lll}
1-\varepsilon^{2}-\varepsilon^{3}+o_{11}\left(\varepsilon^{3}\right) & \varepsilon^{2}+\varepsilon^{3}+o_{12}\left(\varepsilon^{3}\right) & 0 \\
\frac{1}{2} \varepsilon+\frac{1}{2} \varepsilon^{2}-\varepsilon^{3}+o_{21}\left(\varepsilon^{3}\right) & 1-\varepsilon-\varepsilon^{2}+o_{22}\left(\varepsilon^{2}\right) & \frac{1}{2} \varepsilon+\frac{1}{2} \varepsilon^{2}+2 \varepsilon^{3}+o_{23}\left(\varepsilon^{3}\right) \\
\frac{1}{2}+\varepsilon^{2}-\varepsilon^{3}+o_{31}\left(\varepsilon^{3}\right) & \frac{1}{2}-\varepsilon^{2}+\varepsilon^{3}+o_{32}\left(\varepsilon^{3}\right) & 0
\end{array}\right\| .
$$

The $3 \times 3$ matrix of expectations of sojourn times $\left\|e_{i j}(\varepsilon)\right\|$, for the semi-Markov process $\eta^{(\varepsilon)}(t)$, has the following form,

$$
\left\|\begin{array}{lll}
\varepsilon+\varepsilon^{2}+\dot{o}_{11}\left(\varepsilon^{2}\right) & \varepsilon^{3}+\varepsilon^{4}+\dot{o}_{12}\left(\varepsilon^{4}\right) & 0 \\
\varepsilon+\varepsilon^{2}+\dot{o}_{21}\left(\varepsilon^{2}\right) & 1-\varepsilon+\varepsilon^{3}+\dot{o}_{22}\left(\varepsilon^{3}\right) & 2+\varepsilon+\varepsilon^{2}+\dot{o}_{23}\left(\varepsilon^{2}\right) \\
\varepsilon^{-1}+1+\dot{o}_{31}(1) & 2 \varepsilon^{-1}+\varepsilon+\dot{o}_{32}(\varepsilon) & 0
\end{array}\right\|
$$

We assume that conditions $\mathbf{A}-\mathbf{E}$ hold for semi-Markov processes $\eta^{(\varepsilon)}(t)$, i.e. that matrices $\left\|p_{i j}(\varepsilon)\right\|$ and $\left\|e_{i j}(\varepsilon)\right\|$ given in the above relations, can, for every $\varepsilon \in\left(0, \varepsilon_{0}\right]$, serve as, respectively, the matrix of transition probabilities for the corresponding embedded Markov chain and the matrix of expectations of sojourn times.

The matrices of transition probabilities $\left\|p_{i j}(\varepsilon)\right\|$, for the embedded Markov chains $\eta_{n}^{(\varepsilon)}$, and $\left\|p_{i j}(0)\right\|$, for the limiting Markov chain $\eta_{n}^{(0)}$, have, respectively, the following structures,

$$
\left\|\bullet \begin{array}{ll||}
\bullet \bullet \\
\bullet \bullet
\end{array}\right\| \text { and }\left\|\begin{array}{ccc}
1 & 0 & 0 \\
0 & 1 & 0 \\
\frac{1}{2} & \frac{1}{2} & 0
\end{array}\right\|
$$

where symbol $\bullet$ indicates positions of positive elements in matrices $\left\|p_{i j}(\varepsilon)\right\|, \varepsilon \in\left(0, \varepsilon_{0}\right]$.

The phase space $\mathbb{X}$ is one class of communicative states for the embedded Markov chain $\eta_{n}^{(\varepsilon)}$, for every $\varepsilon \in\left(0, \varepsilon_{0}\right]$, while it consists of two closed classes of communicative states $\mathbb{X}_{1}=\{1\}, \mathbb{X}_{2}=\{2\}$ and the class of transient states $\mathbb{X}_{3}=\{3\}$, for the limiting Markov chain $\eta_{n}^{(0)}$.

By excluding the state 1 from the phase space $\mathbb{X}$ and using the algorithm described in Section 10, we construct the reduced semi-Markov processes ${ }_{1} \eta^{(\varepsilon)}(t)$, with the phase space $1 \mathbb{X}=\{2,3\}$. Conditions $\mathbf{A}-\mathbf{E}$ hold for these reduced semi-Markov processes. The corresponding transition sets are ${ }_{1} \mathbb{Y}_{2}=\{2,3\}$ and ${ }_{1} \mathbb{Y}_{3}=\{2\}$. By applying the algorithms described in Lemma 8 and Theorems 2 and 3, we can compute the $2 \times 2$ matrices $\left\|_{1} p_{i j}(\varepsilon)\right\|$ and $\left\|_{1} e_{i j}(\varepsilon)\right\|$. These matrices take the following forms,

$$
\left\|\begin{array}{ll}
1-\frac{1}{2} \varepsilon-\frac{1}{2} \varepsilon^{2}+{ }_{1} o_{22}\left(\varepsilon^{2}\right) & \frac{1}{2} \varepsilon+\frac{1}{2} \varepsilon^{2}+2 \varepsilon^{3}+{ }_{1} o_{23}\left(\varepsilon^{3}\right) \\
1 & 0
\end{array}\right\|,
$$

and

$$
\left\|\begin{array}{ll}
\frac{3}{2}+\frac{1}{2} \varepsilon+{ }_{1} \dot{o}_{22}(\varepsilon) & 2+\varepsilon+\varepsilon^{2}+{ }_{1} \dot{o}_{23}\left(\varepsilon^{2}\right) \\
\frac{7}{2} \varepsilon^{-1}+1+{ }_{1} \dot{o}_{32}(1) & 0
\end{array}\right\| .
$$

By excluding the state 2 from the reduced phase space ${ }_{1} \mathbb{X}=\{2,3\}$, we construct the "final" reduced semi-Markov processes $\langle 1,2\rangle \eta^{(\varepsilon)}(t)$, with the one-state phase space $\langle 1,2\rangle \mathbb{X}=\{3\}$. Conditions $\mathbf{A}-\mathbf{E}$ also hold for these semi-Markov processes. The corresponding transition set $\langle 1,2\rangle \mathbb{Y}=\{3\}$. The transition probability $\langle 1,2\rangle p_{33}(\varepsilon) \equiv 1$. By applying the algorithms described in Theorem 4, we can also compute the Laurent asymptotic expansion for the expected return time, $\langle 1,2\rangle e_{33}(\varepsilon)=E_{33}(\varepsilon)=\frac{21}{2} \varepsilon^{-1}-3+\ddot{o}_{33}(1)$. The Laurent asymptotic expansion for the expected sojourn time $e_{3}(\varepsilon)=e_{31}(\varepsilon)+e_{32}(\varepsilon)+e_{33}(\varepsilon)$, 
obtained with the use of the multiple summation rule given in Lemma 3, has the form, $e_{3}(\varepsilon)=3 \varepsilon^{-1}+1+\dot{o}_{3}(1)$. Finally, the algorithm described in Theorem 5 gives the following asymptotic expansion, for the stationary probability,

$$
\pi_{3}(\varepsilon)=\frac{e_{3}(\varepsilon)}{E_{33}(\varepsilon)}=\frac{3 \varepsilon^{-1}+1+\dot{o}_{3}(1)}{\frac{21}{2} \varepsilon^{-1}-3+\ddot{o}_{33}(1)}=\frac{2}{7}+\frac{26}{147} \varepsilon+o_{3}(\varepsilon) .
$$

Also, by excluding the state 3 from the reduced phase space $1 \mathbb{X}=\{2,3\}$ and applying the algorithms described in Theorem 4, we can compute the Laurent asymptotic expansion for the expected return time $\langle 1,3\rangle e_{22}(\varepsilon)=E_{22}(\varepsilon)=\frac{21}{4}+\frac{15}{4} \varepsilon+\ddot{o}_{22}(\varepsilon)$. In this case, the asymptotic expansion for the expected sojourn time $e_{2}(\varepsilon)=3+\varepsilon+2 \varepsilon^{2}+\dot{o}_{2}\left(\varepsilon^{2}\right)$, and the algorithm described in Theorem 5 gives the following asymptotic expansion for the stationary probability,

$$
\pi_{2}(\varepsilon)=\frac{e_{2}(\varepsilon)}{E_{22}(\varepsilon)}=\frac{3+\varepsilon+2 \varepsilon^{2}+\dot{o}_{2}\left(\varepsilon^{2}\right)}{\frac{21}{4}+\frac{15}{4} \varepsilon+\ddot{o}_{22}(\varepsilon)}=\frac{4}{7}-\frac{32}{147} \varepsilon+o_{2}(\varepsilon) .
$$

As far as the stationary probability $\pi_{1}(\varepsilon)$ is concerned, the corresponding asymptotic expansion can be found using the identity, $\pi_{1}(\varepsilon)=1-\pi_{2}(\varepsilon)-\pi_{3}(\varepsilon), \varepsilon \in\left(0, \varepsilon_{0}\right]$, and the operational rules for asymptotic expansions given in Lemma 3 . This yields the asymptotic expansion,

$$
\pi_{1}(\varepsilon)=\frac{1}{7}+\frac{6}{147} \varepsilon+o_{1}(\varepsilon)
$$

Alternatively, the exclusion of states from the phase space $\mathbb{X}$ in the opposite order, first state 3 and then state 2 or 1, and the use of the algorithms described in Lemma 8 and Theorems $2-4$ yield the asymptotic expansions for the expected return times $\langle 3,2\rangle e_{11}(\varepsilon)=$ $E_{11}(\varepsilon)=7 \varepsilon+\frac{15}{3} \varepsilon^{2}+\ddot{o}_{11}\left(\varepsilon^{2}\right)$ and $\langle 3,1\rangle e_{22}(\varepsilon)=E_{22}(\varepsilon)=\frac{21}{4}+\frac{15}{4} \varepsilon+\ddot{o}_{22}(\varepsilon)$. Then, the algorithm described in Theorem $5_{*}$ yields the same asymptotic expansions for stationary probabilities $\pi_{1}(\varepsilon)=\frac{e_{1}(\varepsilon)}{E_{11}(\varepsilon)}=\frac{\varepsilon+\varepsilon^{2}+\dot{o}_{1}\left(\varepsilon^{2}\right)}{7 \varepsilon+\frac{15}{3} \varepsilon^{2}+\ddot{o}_{11}\left(\varepsilon^{2}\right)}=\frac{1}{7}+\frac{6}{147} \varepsilon+o_{1}(\varepsilon)$ and $\pi_{2}(\varepsilon)=\frac{e_{2}(\varepsilon)}{E_{22}(\varepsilon)}=$ $\frac{3+\varepsilon+2 \varepsilon^{2}+\dot{o}_{2}\left(\varepsilon^{2}\right)}{\frac{21}{4}+\frac{15}{4} \varepsilon+\ddot{o}_{22}(\varepsilon)}=\frac{4}{7}-\frac{32}{147} \varepsilon+o_{2}(\varepsilon)$.

Note that the Laurent asymptotic expansion for the expectation $E_{22}(\varepsilon)$ and, in sequel, the Taylor asymptotic expansion for the stationary probability $\pi_{2}(\varepsilon)$ are invariant with respect to the choice the sequence of states $\langle 1,3\rangle$ or $\langle 3,1\rangle$ for sequential exclusion from the phase space $\mathbb{X}$. This is consistent with the corresponding invariance statements formulated in Theorems 4 and 5.

The coefficients of the asymptotic expansions $\pi_{i}(\varepsilon)=c_{i}[0]+c_{i}[1] \varepsilon+o_{i}(\varepsilon), i=1,2,3$ given above satisfy relations, $c_{1}[0]+c_{2}[0]+c_{3}[0]=1$ and $c_{1}[1]+c_{2}[1]+c_{3}[1]=0$. This is consistent with the corresponding statement of Theorem 5.

In the example presented above, we did not trace the explicit formulas for remainders $o_{1}(\varepsilon), o_{2}(\varepsilon)$ and $o_{3}(\varepsilon)$. However, according to the corresponding statement in Theorem 5, these remainders are connected by the following identity $o_{1}(\varepsilon)+o_{2}(\varepsilon)+o_{3}(\varepsilon) \equiv 0$.

We would like also to explain an unexpected, in some sense, asymptotic behavior of stationary probabilities $\pi_{i}(\varepsilon)$, in the above example. As a matter of fact, states 1 and 2 are asymptotically absorbing states with non-absorption probabilities of different order, respectively, $O\left(\varepsilon^{2}\right)$ and $O(\varepsilon)$. While, state 3 is a transient asymptotically non-absorbing state. This, seems, should cause convergence of the stationary probability $\pi_{1}(\varepsilon)$ to 1 and the stationary probabilities $\pi_{2}(\varepsilon)$ and $\pi_{3}(\varepsilon)$ to 0 as $\varepsilon \rightarrow 0$, with different rates of convergence. This, however, does not take place, and all three probabilities converge to non-zero limits. 
This is because of the expected sojourn times $e_{1}(\varepsilon), e_{2}(\varepsilon)$ and $e_{3}(\varepsilon)$ have orders, respectively, $O(\varepsilon), O(1)$ and $O\left(\varepsilon^{-1}\right)$. These expectations compensate absorption effects for states 1,2 and 3.

In the above example, computations of explicit upper bounds for remainders in the asymptotic expansions for stationary probabilities $\pi_{1}(\varepsilon), \pi_{2}(\varepsilon)$ and $\pi_{3}(\varepsilon)$ can also be realized in the case, where conditions $\mathbf{D}^{\prime}$ and $\mathbf{E}^{\prime}$ hold instead of conditions $\mathbf{D}$ and $\mathbf{E}$. We, however, omit this presentation, in order to escape overloading the paper by technical numerical computations.

In conclusion, we would like also to mention that results related to applications of asymptotic expansions for expectations of hitting times and stationary distributions for perturbed semi-Markov processes and Markov chains to the asymptotical analysis of perturbed queuing and control systems, reliability models, stochastic networks and modes of bio-stochastic systems can be found in the works referred in Part 1 of this paper. Also, a survey and a comprehensive bibliography of works in related areas can be found in paper Silvestrov and Silvestrov (2016a).

\section{References}

Hörmander L (1990) An introduction to complex analysis in several variables. North-Holland Mathematical Library, vol 7, 3rd edn. North-Holland, Amsterdam, p xii+254. (3rd edition of An introduction to complex analysis in several variables, D. Van Nostrand, Princeton, 1966, x+208 pp)

Markushevich AI (1985) Theory of functions of a complex variable. Vol I, II, III, 2nd edn. Chelsea, New York, p xxiii+1138. (English edition: Theory of analytic functions. GITTL, Moscow, 1950)

Silvestrov D, Silvestrov S (2017) Asymptotic expansions for stationary distributions of nonlinearly perturbed semi-Markov processes. 1. Method Comp Appl Probab. https://doi.org/10.1007/s11009-017-9605-0

Silvestrov D, Silvestrov S (2016a) Asymptotic expansions for stationary distributions of perturbed semiMarkov processes. In: Silvestrov S, Rančić M (eds) Engineering mathematics II. Algebraic, stochastic and analysis structures for networks, data classification and optimization. Chapter 10. Springer proceedings in mathematics \& statistics, vol 179. Springer, Cham, pp 151-222

Silvestrov D, Silvestrov S (2016b) Asymptotic expansions for stationary distributions of nonlinearly perturbed semi-Markov processes. I, II. Part I: arXiv:1603.04734, 30 pp. Part II: arXiv:1603.04743, $33 \mathrm{pp}$ 